\title{
The statistical properties of host load ${ }^{1}$
}

\author{
Peter A. Dinda \\ Carnegie Mellon University, 5000 Forbes Avenue, \\ Pittsburgh, PA 15213, USA \\ Tel.: +1 412268 3077; Fax: +1 412268 5576; \\ E-mail: pdinda@cs.cmu.edu
}

Understanding how host load changes over time is instrumental in predicting the execution time of tasks or jobs, such as in dynamic load balancing and distributed soft real-time systems. To improve this understanding, we collected weeklong, $1 \mathrm{~Hz}$ resolution traces of the Digital Unix 5 second exponential load average on over 35 different machines including production and research cluster machines, compute servers, and desktop workstations. Separate sets of traces were collected at two different times of the year. The traces capture all of the dynamic load information available to userlevel programs on these machines. We present a detailed statistical analysis of these traces here, including summary statistics, distributions, and time series analysis results. Two significant new results are that load is self-similar and that it displays epochal behavior. All of the traces exhibit a high degree of self-similarity with Hurst parameters ranging from 0.73 to 0.99 , strongly biased toward the top of that range. The traces also display epochal behavior in that the local frequency content of the load signal remains quite stable for long periods of time (150-450 s mean) and changes abruptly at epoch boundaries. Despite these complex behaviors, we have found that relatively simple linear models are sufficient for short-range host load prediction.

Keywords: host load properties, host load prediction, selfsimilarity, long-range dependence, epochal behavior

\footnotetext{
${ }^{1}$ Effort sponsored in part by the Advanced Research Projects Agency and Rome Laboratory, Air Force Materiel Command, USAF, under agreement number F30602-96-1-0287, in part by the National Science Foundation under Grant CMS-9318163, and in part by a grant from the Intel Corporation. The U.S. Government is authorized to reproduce and distribute reprints for Governmental purposes notwithstanding any copyright annotation thereon. The views and conclusions contained herein are those of the authors and should not be interpreted as necessarily representing the official policies or endorsements, either expressed or implied, of the Advanced Research Projects Agency, Rome Laboratory, or the U.S. Government.
}

\section{Introduction}

The distributed computing environments to which most users have access consist of a collection of loosely interconnected hosts running vendor operating systems. Tasks are initiated independently by users and are scheduled locally by a vendor supplied operating system; there is no global scheduler that controls access to the hosts. As users run their jobs the computational load on the individual hosts changes over time.

Deciding how to map computations to hosts in systems with such dynamically changing loads (what we will call the mapping problem) is a basic problem that arises in a number of important contexts, such as dynamically load-balancing the tasks in a parallel program $[24,1,26]$, and scheduling tasks to meet deadlines in a distributed soft real-time system $[15,22,23,17]$.

Host load has a significant effect on running time. Indeed, the running time of a compute bound task is directly related to the average load it encounters during execution. Determining a good mapping of a task requires a prediction, either implicit or explicit, of the load on the prospective remote hosts to which the task could be mapped. Making such predictions demands an understanding of the qualitative and quantitative properties of load on real systems. If the tasks to be mapped are short, this understanding of load should extend to correspondingly fine resolutions. Unfortunately, to date there has been little work on characterizing the properties of load at fine resolutions. The available studies concentrate on understanding functions of load, such as availability [21] or job durations $[8,18$, 11]. Furthermore, they deal with the coarse grain behavior of load - how it changes over minutes, hours and days.

This paper is a first step to a better understanding the properties of load on real systems at fine resolutions. We collected week-long, $1 \mathrm{~Hz}$ resolution traces of the Digital Unix load average (specifically, an exponential average with a five second time constant) on over 35 different machines that we classify as production and research cluster machines, compute servers, or desktop workstations. We collected two sets of such traces at different times of the year. The $1 \mathrm{~Hz}$ sample rate 
is sufficient to capture all of the dynamic load information that is available to user-level programs running on these machines. In this paper, we present a detailed statistical analysis of both sets of traces and contemplate the implications of the properties we find for the mapping problem. An earlier version of this paper [6], concentrated on the first set of traces.

The basic question is whether load traces that might seem at first glance to be random and unpredictable might have structure that could be exploited by a mapping algorithm. Our results suggest that load traces do indeed have some structure in the form of clearly identifiable properties. In essence, our results characterize how load varies, which should be of interest not only to developers of mapping and prediction algorithms, but also to those who need to generate realistic synthetic loads in simulators or to those doing analytic work. Here is a summary of our results and their implications:

(1) The traces exhibit low means but very high standard deviations and maximums. Relatively few of the traces had mean loads of 1.0 or more. The standard deviation is typically at least as large as the mean, while the maximums can be as much as two orders of magnitude larger. The implication is that these machines have plenty of cycles to spare to execute jobs, but the execution time of these jobs will vary drastically.

(2) Standard deviation and maximum, which are absolute measures of variation, are positively correlated with the mean, so a machine with a high mean load will also tend to have a large standard deviation and maximum. However, these measures do not grow as quickly as the mean, so their corresponding relative measures actually shrink as the mean increases. The implication is that if the mapping problem assumes a relative metric, it may not be unreasonable to use the host with higher mean load.

(3) The traces have complex, rough, and often multimodal distributions that are not well fitted by analytic distributions such as the normal or exponential distributions. Even for the traces which exhibit unimodally distributed load, the normal distribution's tail is too short while the exponential distribution's tail is too long. The implication is that modeling and simulation that assumes convenient analytical load distributions may be flawed.

(4) Time series analysis of the traces shows that load is strongly correlated over time. The autocorrelation function typically decays very slowly while the periodogram shows a broad, almost noise-like combination of all frequency components. An important implication is that history-based load prediction schemes seem very feasible. However, the complex frequency domain behavior suggests that linear modeling schemes may have difficulty. From a modeling point of view, it is clearly important that these dependencies between successive load measurements are captured.

(5) The traces are self-similar. Their Hurst parameters range from 0.73 to 0.99 , with a strong bias toward the top of that range. This tells us that load varies in complex ways on all time scales and is long term dependent. This has several important implications. First, smoothing load by averaging over an interval results in much smaller decreases in variance than if load were not long range dependent. Variance decays with increasing interval length $m$ and Hurst parameter $H$ as $m^{2 H-2}$. This is $m^{-1.0}$ for signals without long range dependence and $m^{-0.54}$ to $m^{-0.02}$ for the range of $H$ we measured. This suggests that task migration in the face of adverse load conditions may be preferable to waiting for the adversity to be ameliorated over the long term. The self-similarity result also suggests certain modeling approaches, such as fractional ARIMA models $[12,10,3]$ which can capture this property.

(6) The traces display epochal behavior. The local frequency content of the load signal remains quite stable for long periods of time (150-450 s mean) and changes abruptly at the boundaries of such epochs. This suggests that the problem of predicting load may be able to be decomposed into a sequence of smaller subproblems.

After completing this study, we evaluated linear models for predicting host load using the traces, finding that relatively simple autoregressive models are sufficient for short range host load prediction [7].

\section{Measurement methodology}

The load on a Unix system at any given instant is the number of processes that are running or are ready to run, which is the length of the ready queue maintained by the scheduler. The kernel samples the length of the the ready queue at some rate and exponentially averages some number of previous samples to produce a load average which can be accessed from a user program. The specific Unix system we used was Digital Unix (DUX).

Unlike many Unix implementations, which exponentially average with a time constant of one minute at the finest, DUX uses a time constant of five seconds. This small time constant allows us to capture considerably more of the dynamics of load than would have 
been possible on other Unix implementations, and it minimizes the effect of phantom correlations due to the exponential filter. Interestingly, directly sampling the length of the ready queue, which we tried on Windows NT, does not provide much useful information because it is impossible to sample the queue fast enough from a user process.

We developed a small tool to sample the DUX load average at one second intervals and log the resulting time series to a data file. The $1 \mathrm{~Hz}$ sample rate was arrived at by subjecting DUX systems to varying loads and sampling at progressively higher rates to determine the rate at which DUX actually updated the value. DUX updates the value at a rate of $1 / 2 \mathrm{~Hz}$, thus we chose a $1 \mathrm{~Hz}$ sample rate by the Nyquist criterion. This choice of sample rate means we capture all of the dynamic load information the operating system makes available to user programs. We ran this trace collection tool on 39 hosts belonging to the Computing, Media, and Communication Laboratory (CMCL) at CMU and the Pittsburgh Supercomputing Center (PSC) for slightly more than one week in late August, 1997. A second set of week-long traces was acquired on almost exactly the same set of machines (35 machines total) in late February and early March, 1998. The results of the statistical analysis were similar for the two sets of traces.

All of the hosts in the August, 1997 set were DEC Alpha DUX machines, running either DUX 3.2 or 4.0 and they form four classes:

- Production Cluster: 13 hosts of the PSC's "Supercluster", including two front-end machines (axpfea, axpfeb), four interactive machines (axp0 through axp3), and seven batch machines scheduled by a DQS [16] variant (axp4 through axp10).

- Research Cluster: eight machines in an experimental cluster in the CMCL (manchester-1 through manchester-8).

- Compute servers: two high performance large memory machines used by the CMCL group as compute servers for simulations and the like (mojave and sahara).

- Desktops: 16 desktop workstations owned by members of the CMCL (aphrodite through zeno).

The same hosts were used for the March, 1998 traces, with the following exceptions:

- Production Cluster: axp9 was replaced by axp11 due to hardware failures.

- Desktops: argus, asclepius, bruce, cobain, darryl, and hestia were replaced by belushi and loman due to hardware upgrades.
Tables 1 and 2 provide additional details of the individual August, 1997 and March, 1998 traces. The author will be happy to provide the traces to any interested readers.

\section{Statistical analysis}

We analyzed the individual load traces using summary statistics, histograms, fitting of analytic distributions, and time series analysis. The picture that emerges is that load varies over a wide range in very complex ways. Load distributions are rough and frequently multi-modal. Even traces with unimodal histograms are not well fitted by common analytic distributions, which have tails that are either too short or too long. Time series analysis shows that load is strongly correlated over time, but also has complex, almost noise-like frequency domain behavior.

We summarized each of our load traces in terms of our statistical measures and computed their correlations to determine how the measures are related. Table 3(a) contains the correlations for the August, 1997 set while Table 3(b) contains the correlations for the March, 1998 set. Unless otherwise noted, all figures in the paper similarly present independent results for the two sets of traces. Each cell of Table 3 is the correlation coefficient (CC) between the row measure and the column measure, computed over the the load traces in the set. We will refer back to the highlighted values (where the absolute correlations are greater than 0.3 ) throughout the paper. It is important to note that these cross correlations can serve as a basis for clustering load traces into rough equivalence classes.

\section{Summary statistics}

Summarizing each load trace in terms of its mean, standard deviation, and maximum and minimum illustrates the extent to which load varies. Fig. 1 shows the mean load and the $+/-$ one standard deviation points for each of the traces. As we might expect, the mean load on desktop machines is significantly lower than on other machines. However, we can also see a lack of uniformity within each class, despite the long duration of the traces. This is most clear among the Production Cluster machines, where fewer than half of the machines seem to be doing most of the work. This lack of uniformity even over long time scales shows clear opportunity for load balancing or resource management systems. 
Table 1

Details of the August, 1997 traces

\begin{tabular}{|c|c|c|c|}
\hline Hostname & Start time & Days & Samples \\
\hline \multicolumn{4}{|c|}{ Production cluster } \\
\hline axp0.psc & Tue Aug 12 21:29:12 EDT 1997 & 15.00 & 1296000 \\
\hline axp1.psc & Tue Aug 12 21:30:09 EDT 1997 & 14.00 & 1209600 \\
\hline axp2.psc & Tue Aug 12 21:30:53 EDT 1997 & 14.00 & 1209600 \\
\hline axp3.psc & Tue Aug 12 21:31:13 EDT 1997 & 14.00 & 1209600 \\
\hline axp4.psc & Tue Aug 12 21:31:12 EDT 1997 & 14.00 & 1209600 \\
\hline axp5.psc & Tue Aug 12 21:31:47 EDT 1997 & 14.00 & 1209600 \\
\hline axp6.psc & Tue Aug 12 21:31:15 EDT 1997 & 15.00 & 1296000 \\
\hline axp7.psc & Tue Aug 12 20:51:19 EDT 1997 & 13.00 & 1123200 \\
\hline axp8.psc & Tue Aug 12 21:31:19 EDT 1997 & 14.00 & 1209600 \\
\hline axp9.psc & Tue Aug 12 21:31:45 EDT 1997 & 14.00 & 1209600 \\
\hline axp10.psc & Tue Aug 12 21:31:21 EDT 1997 & 14.00 & 1209600 \\
\hline axpfea.psc & Sat Aug 16 14:44:29 EDT 1997 & 13.00 & 1123200 \\
\hline axpfeb.psc & Sat Aug 16 14:44:55 EDT 1997 & 12.00 & 1036800 \\
\hline \multicolumn{4}{|c|}{ Research cluster } \\
\hline manchester-1.cmcl & Sun Aug 17 19:41:10 EDT 1997 & 3.92 & 338400 \\
\hline manchester-2.cmcl & Sun Aug 17 19:41:09 EDT 1997 & 4.00 & 345600 \\
\hline manchester-3.cmcl & Sun Aug 17 19:41:13 EDT 1997 & 3.96 & 342000 \\
\hline manchester-4.cmcl & Sun Aug 17 19:41:10 EDT 1997 & 4.00 & 345600 \\
\hline manchester-5.cmcl & Sun Aug 17 19:41:09 EDT 1997 & 4.04 & 349200 \\
\hline manchester-6.cmcl & Sun Aug 17 19:41:09 EDT 1997 & 4.08 & 352800 \\
\hline manchester-7.cmcl & Sun Aug 17 19:41:10 EDT 1997 & 4.00 & 345600 \\
\hline manchester-8.cmcl & Sun Aug 17 19:41:10 EDT 1997 & 4.00 & 345600 \\
\hline \multicolumn{4}{|c|}{ Compute servers } \\
\hline mojave.cmcl & Sun Aug 17 19:41:11 EDT 1997 & 4.04 & 349200 \\
\hline sahara.cmcl & Sun Aug 17 19:41:11 EDT 1997 & 4.00 & 345600 \\
\hline \multicolumn{4}{|c|}{ Desktops } \\
\hline aphrodite.nectar & Sun Aug 17 19:41:12 EDT 1997 & 4.00 & 345600 \\
\hline argus.nectar & Sun Aug 17 19:41:17 EDT 1997 & 4.04 & 349200 \\
\hline asbury-park.nectar & Sun Aug 17 19:41:11 EDT 1997 & 4.00 & 345600 \\
\hline asclepius.nectar & Sun Aug 17 19:41:07 EDT 1997 & 4.08 & 352800 \\
\hline bruce.nectar & Sun Aug 17 19:41:10 EDT 1997 & 3.92 & 338400 \\
\hline cobain.nectar & Sun Aug 17 19:41:12 EDT 1997 & 4.04 & 349200 \\
\hline darryl.nectar & Sun Aug 17 19:41:32 EDT 1997 & 1.71 & 147600 \\
\hline hawaii.cmcl & Sun Aug 17 19:41:11 EDT 1997 & 2.63 & 226800 \\
\hline hestia.nectar & Sun Aug 17 19:41:12 EDT 1997 & 4.00 & 345600 \\
\hline newark.cmcl & Sun Aug 17 19:41:13 EDT 1997 & 4.00 & 345600 \\
\hline pryor.nectar & Sun Aug 17 19:41:13 EDT 1997 & 1.71 & 147600 \\
\hline rhea.nectar & Sun Aug 17 19:41:11 EDT 1997 & 4.00 & 345600 \\
\hline rubix.mc & Sun Aug 17 19:41:13 EDT 1997 & 4.00 & 345600 \\
\hline themis.nectar & Sun Aug 17 19:41:09 EDT 1997 & 4.00 & 345600 \\
\hline uranus.nectar & Sun Aug 17 19:41:13 EDT 1997 & 4.00 & 345600 \\
\hline zeno.nectar & Sun Aug 17 19:41:13 EDT 1997 & 4.08 & 352800 \\
\hline
\end{tabular}


Table 2

Details of the March, 1998 traces

\begin{tabular}{|c|c|c|c|}
\hline Hostname & Start time & Days & Samples \\
\hline \multicolumn{4}{|c|}{ Production cluster } \\
\hline axp0.psc & Wed Feb 25 17:34:26 EST 1998 & 12.08 & 1043400 \\
\hline axp1.psc & Wed Feb 25 17:34:25 EST 1998 & 12.08 & 1043400 \\
\hline axp2.psc & Wed Feb 25 17:34:25 EST 1998 & 12.08 & 1043400 \\
\hline axp3.psc & Wed Feb 25 17:34:26 EST 1998 & 12.03 & 1039400 \\
\hline axp4.psc & Wed Feb 25 17:34:27 EST 1998 & 12.06 & 1041900 \\
\hline axp5.psc & Wed Feb 25 17:34:27 EST 1998 & 12.03 & 1039700 \\
\hline axp6.psc & Wed Feb 25 17:34:27 EST 1998 & 12.08 & 1043400 \\
\hline axp7.psc & Wed Feb 25 17:34:27 EST 1998 & 12.01 & 1037700 \\
\hline axp8.psc & Wed Feb 25 17:34:28 EST 1998 & 12.06 & 1041800 \\
\hline axp10.psc & Wed Feb 25 17:34:28 EST 1998 & 12.03 & 1039700 \\
\hline axp11.psc & Wed Feb 25 17:16:20 EST 1998 & 12.03 & 1039800 \\
\hline axpfea.psc & Wed Feb 25 17:18:01 EST 1998 & 12.08 & 1043800 \\
\hline axpfeb.psc & Wed Feb 25 17:23:34 EST 1998 & 12.0 & 1043400 \\
\hline \multicolumn{4}{|c|}{ Research cluster } \\
\hline manchester-1.cmcl & Wed Feb 25 20:42:30 EST 1998 & 8.36 & 721900 \\
\hline manchester-2.cmcl & Wed Feb 25 20:42:23 EST 1998 & 8.36 & 721900 \\
\hline manchester-3.cmcl & Wed Feb 25 20:42:23 EST 1998 & 8.36 & 721900 \\
\hline manchester-4.cmcl & Wed Feb 25 20:42:29 EST 1998 & 8.35 & 721300 \\
\hline manchester-5.cmcl & Wed Feb 25 20:42:27 EST 1998 & 8.36 & 721900 \\
\hline manchester-6.cmcl & Wed Feb 25 20:42:30 EST 1998 & 8.36 & 721900 \\
\hline manchester-7.cmcl & Wed Feb 25 20:42:24 EST 1998 & 8.35 & 721800 \\
\hline manchester-8.cmcl & Wed Feb 25 20:42:26 EST 1998 & 8.35 & 721800 \\
\hline \multicolumn{4}{|c|}{ Compute servers } \\
\hline mojave.cmcl & Wed Feb 25 20:42:31 EST 1998 & 5.31 & 458800 \\
\hline sahara.cmcl & Wed Feb 25 20:42:34 EST 1998 & 8.34 & 721300 \\
\hline \multicolumn{4}{|c|}{ Desktops } \\
\hline aphrodite & Wed Feb 25 20:42:17 EST 1998 & 8.36 & 722000 \\
\hline asbury-park & Wed Feb 25 20:42:23 EST 1998 & 5.90 & 509400 \\
\hline belushi & Wed Feb 25 20:42:28 EST 1998 & 7.77 & 671400 \\
\hline hawaii & Wed Feb 25 20:42:18 EST 1998 & 8.36 & 722000 \\
\hline loman & Wed Feb 25 20:42:34 EST 1998 & 8.36 & 721900 \\
\hline newark.cmcl & Wed Feb 25 20:42:29 EST 1998 & 8.36 & 722200 \\
\hline pryor.nectar & Wed Feb 25 20:42:24 EST 1998 & 8.36 & 722100 \\
\hline rhea.nectar & Wed Feb 25 21:12:17 EST 1998 & 10.19 & 880600 \\
\hline rubix.mc & Wed Feb 25 20:42:24 EST 1998 & 1.81 & 156400 \\
\hline themis.nectar & Wed Feb 25 20:42:29 EST 1998 & 4.94 & 426900 \\
\hline uranus.nectar & Wed Feb 25 20:42:33 EST 1998 & 8.36 & 722000 \\
\hline zeno.nectar & Wed Feb 25 20:42:26 EST 1998 & 6.23 & 537900 \\
\hline
\end{tabular}


Table 3

Correlation coefficients (CCs) between all of the discussed statistical properties

(a) Correlations for August, 1997 traces

\begin{tabular}{|c|c|c|c|c|c|c|c|c|c|c|}
\hline & $\begin{array}{l}\text { Mean } \\
\text { Load }\end{array}$ & $\begin{array}{l}\text { Sdev } \\
\text { Load }\end{array}$ & $\begin{array}{l}\text { COV } \\
\text { Load }\end{array}$ & $\begin{array}{l}\text { Max } \\
\text { Load }\end{array}$ & $\begin{array}{c}\text { Max/Mean } \\
\text { Load }\end{array}$ & $\begin{array}{l}\text { Mean } \\
\text { Epoch }\end{array}$ & $\begin{array}{c}\text { Sdev } \\
\text { Epoch }\end{array}$ & $\begin{array}{l}\text { COV } \\
\text { Epoch }\end{array}$ & $\begin{array}{l}\text { Hurst } \\
\text { Param }\end{array}$ & Entropy \\
\hline Mean Load & 1.00 & & & & & & & & & \\
\hline Sdev Load & 0.53 & 1.00 & & & & & & & & \\
\hline COV Load & -0.49 & -0.22 & 1.00 & & & & & & & \\
\hline Max Load & 0.60 & 0.18 & -0.32 & 1.00 & & & & & & \\
\hline Max/Mean Load & -0.36 & -0.39 & 0.51 & 0.03 & 1.00 & & & & & \\
\hline Mean Epoch & -0.04 & -0.10 & -0.19 & 0.08 & -0.05 & 1.00 & & & & \\
\hline Sdev Epoch & -0.02 & -0.10 & -0.20 & 0.09 & -0.06 & 0.99 & 1.00 & & & \\
\hline COV Epoch & 0.07 & -0.11 & -0.23 & 0.15 & -0.02 & 0.95 & 0.96 & 1.00 & & \\
\hline Hurst Param & 0.45 & 0.58 & -0.21 & 0.03 & -0.49 & 0.08 & 0.10 & 0.18 & 1.00 & \\
\hline Entropy & 0.42 & 0.51 & -0.10 & 0.40 & -0.36 & -0.27 & -0.25 & -0.30 & 0.24 & 1.00 \\
\hline \multicolumn{11}{|c|}{ (b) Correlations for March, 1998 traces } \\
\hline & Mean & Sdev & $\mathrm{COV}$ & $\operatorname{Max}$ & Max/Mean & Mean & Sdev & $\mathrm{COV}$ & Hurst & Entropy \\
\hline & Load & Load & Load & Load & Load & Epoch & Epoch & Epoch & Param & \\
\hline Mean Load & 1.00 & & & & & & & & & \\
\hline Sdev Load & 0.72 & 1.00 & & & & & & & & \\
\hline COV Load & -0.64 & -0.48 & 1.00 & & & & & & & \\
\hline Max Load & 0.43 & 0.11 & -0.25 & 1.00 & & & & & & \\
\hline Max/Mean Load & -0.48 & -0.49 & 0.93 & -0.07 & 1.00 & & & & & \\
\hline Mean Epoch & -0.12 & -0.23 & -0.08 & 0.17 & -0.05 & 1.00 & & & & \\
\hline Sdev Epoch & -0.13 & -0.22 & -0.09 & 0.15 & -0.06 & 0.99 & 1.00 & & & \\
\hline COV Epoch & -0.03 & -0.12 & -0.15 & 0.23 & -0.11 & 0.88 & 0.93 & 1.00 & & \\
\hline Hurst Param & $-\mathbf{0 . 3 0}$ & -0.41 & 0.29 & 0.15 & 0.36 & 0.92 & 0.90 & 0.78 & 1.00 & \\
\hline Entropy & 0.05 & 0.27 & -0.19 & -0.05 & -0.27 & -0.19 & -0.18 & -0.17 & -0.29 & 1.00 \\
\hline
\end{tabular}

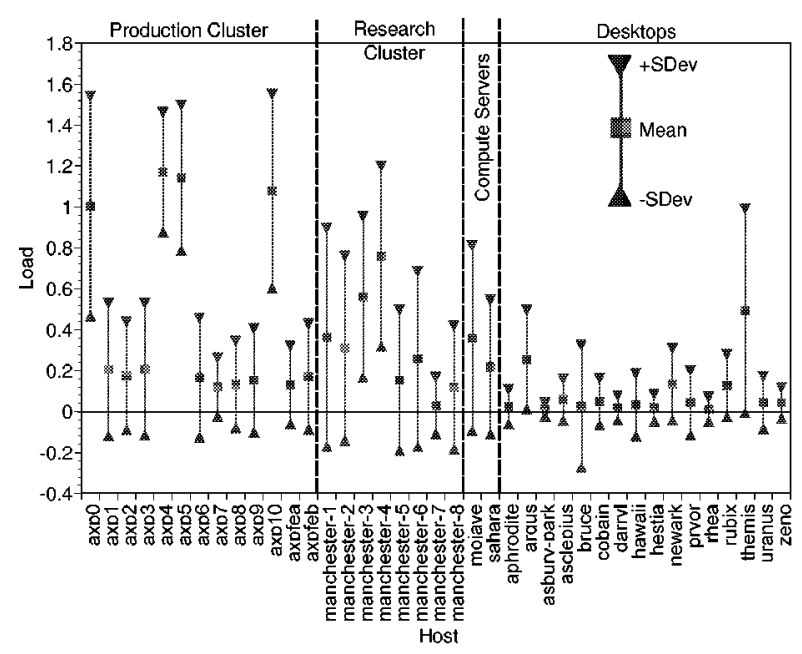

(a)

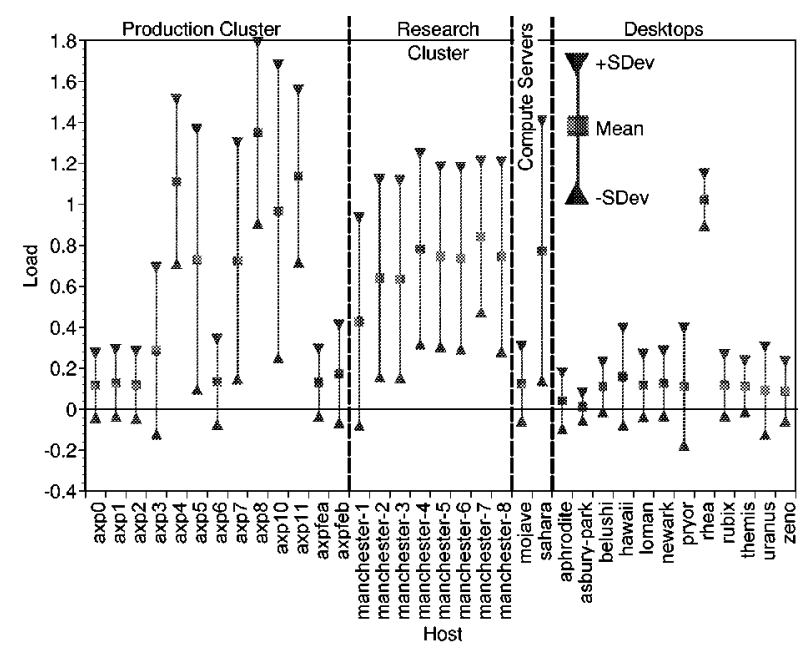

(b)

Fig. 1. Mean load +/- one standard deviation: (a) August, 1997 traces, (b) March, 1998 traces. 


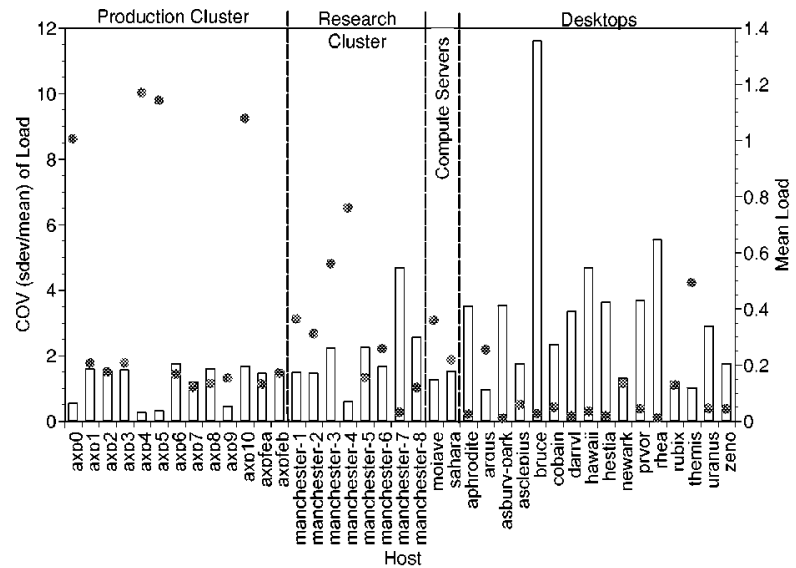

(a)

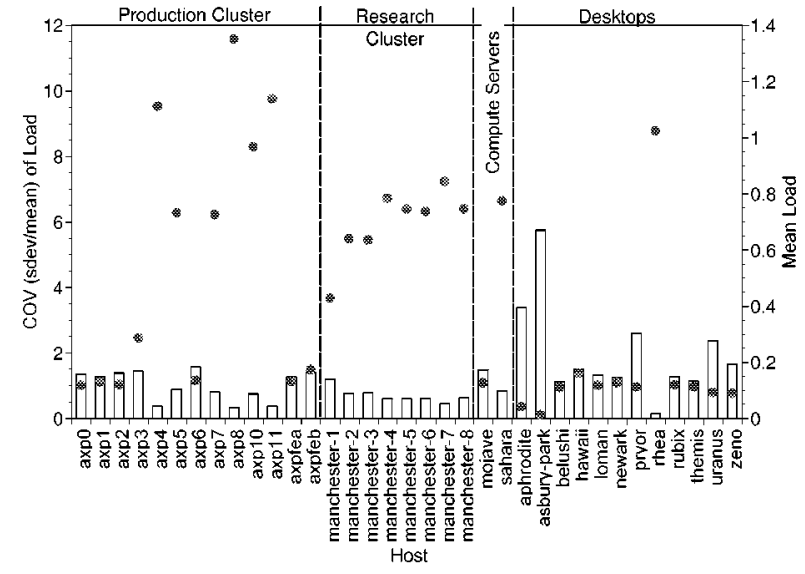

(b)

Fig. 2. COV of load and mean load: (a) August, 1997 traces, (b) March, 1998 traces.

From Fig. 1 we can also see that desktop machines have smaller standard deviations than the other machines. Indeed, the standard deviation, which shows how much load varies in absolute terms, grows with increasing mean load (Table 3 shows $\mathrm{CC}=0.53$ for the 1997 traces and $\mathrm{CC}=0.72$ for the 1998 traces). However, in relative terms, variance shrinks with increasing mean load. This can be seen in Fig. 2, which plots the coefficient of variation (the standard deviation divided by the mean, abbreviated as the COV) and the mean load for each of the load traces. Here we can see that desktop machines, with their smaller mean loads, have large COVs compared to the other classes of machines. The CC between mean load and the COV of load is -0.49 for the 1997 traces and -0.64 for the 1998 traces. It is clear that as load increases, it varies less in relative terms and more in absolute terms.

This difference between absolute and relative behavior also holds true for the maximum load. Fig. 3 shows the minimum, maximum, and mean load for each of the traces. The minimum load is, not surprisingly, zero in almost every case. The maximum load is positively correlated with the mean load (CC $=0.60$ for the 1997 traces and $\mathrm{CC}=0.43$ for the 1998 traces in Table 3). Fig. 4 plots the ratio max/mean and the mean load for each of the traces. It is clear that this relative measure is inversely related to mean load, and Table 3 shows that the CC is -0.36 for the 1997 traces and -0.48 for the 1998 traces. It is also important to notice that while the differences in maximum load between the hosts are rather small (Fig. 3), the differences in the max/mean ratio can be quite large (Fig. 4). Desktops are more surprising machines in relative terms.
With respect to the mapping problem, the implication of the differences between relative and absolute measures of variability is that lightly loaded (low mean load) hosts are not always preferable over heavily loaded hosts. For example, if the performance metric is itself a relative one (that the execution time not vary much relative to the mean execution time, say), then a more heavily loaded host may be preferable.

\section{Distributions}

We next treated each trace as a realization of an independent, identically distributed (IID) stochastic process. Such a process is completely described by its probability distribution function (pdf), which does not change over time. Since we have a vast number of data points for each trace, histograms closely approximate this underlying pdf. We examined the histograms of each of our load traces and fitted normal and exponential distributions to them. To illustrate the following discussion, Fig. 5 shows the histograms of load measurements on (a) axp0 and (b) axp7 on August 19, 1997 (86400 samples each). Axp0 has a high mean load, while axp7 is much more lightly loaded.

Some of the traces, especially those with high mean loads, have multi-modal histograms. Fig. 5(a) is an example of such a multi-modal distribution while Fig. 5(b) shows a unimodal distribution. Typically, the modes are integer multiples of 1.0 (and occasionally 0.5). One explanation for this behavior is that jobs on these machines are for the most part compute bound and thus the ready queue length corresponds to the number of jobs. This seems plausible for the cluster machines, which run scientific workloads for the most 


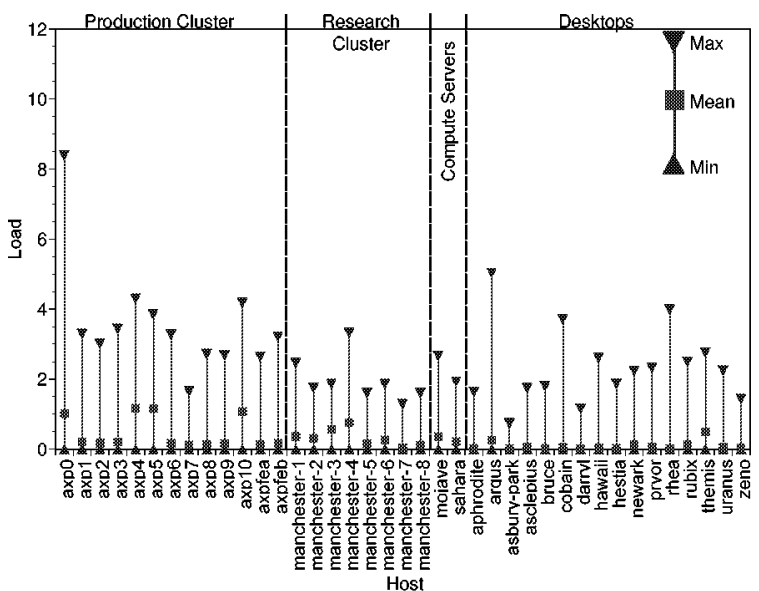

(a)

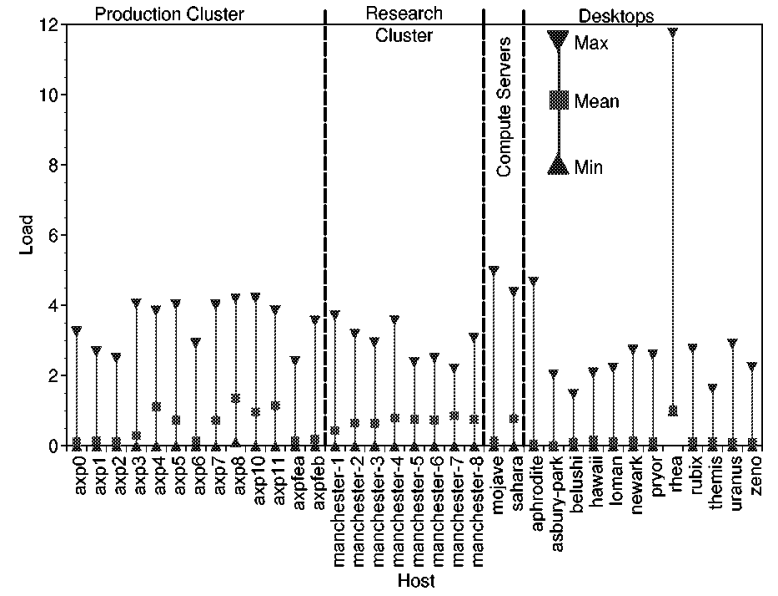

(b)

Fig. 3. Minimum, maximum, and mean load: (a) August, 1997 traces, (b) March, 1998 traces.

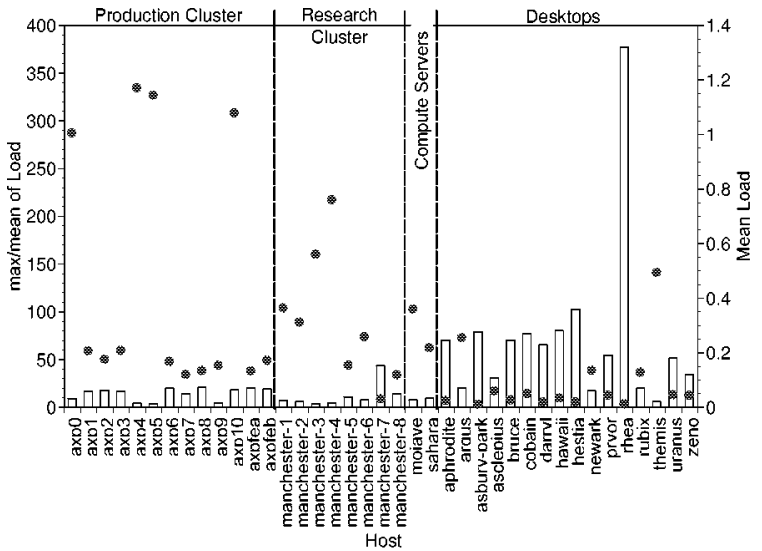

(a)

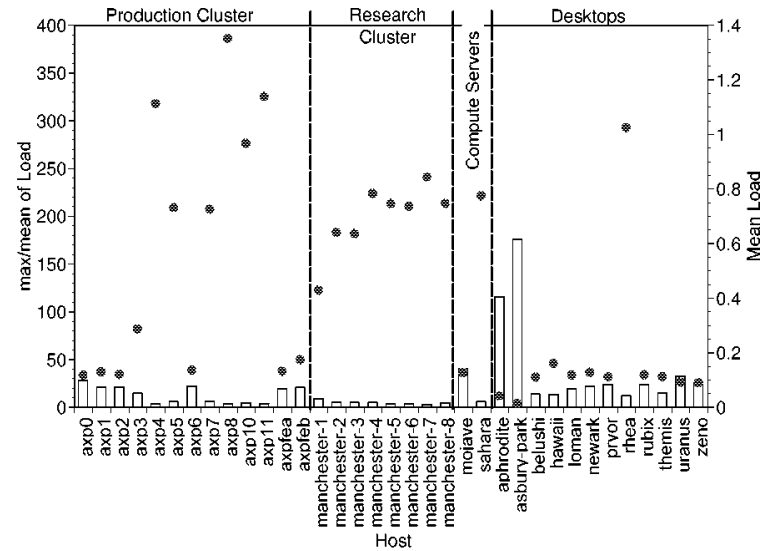

(b)

Fig. 4. Maximum to mean load ratios and mean load: (a) August, 1997 traces, (b) March, 1998 traces.

part. However, such multi-modal distributions were also noticed on the some of the other machines.

The rough appearance of the histograms (consider Fig. 5(b)) is due to the fact that the underlying quantity being measured (ready queue length) is discrete. Load typically takes on 600-3000 unique values in these traces. Shannon's entropy measure [25] indicates that the load traces can be encoded in 1.4 to 8.5 bits per value, depending on the trace. These observations and the histograms suggest that load spends most of its time in one of a small number of levels.

The histograms share very few common characteristics and did not conform well to the analytic distributions we fit to them. Quantile-quantile plots are a powerful way to assess how a distribution fits data (cf. [14], pp. 196-200). The quantiles (the $\alpha$ quantile of a pdf (or histogram) is the value $x$ at which $100 \alpha \%$ of the probability (or data) falls to the left of $x$ ) of the data set are plotted against the quantiles of the hypothetical analytic distribution. Regardless of the choice of parameters, the plot will be linear if the data fits the distribution.

We fitted normal and exponential distributions to each of the load traces. The fits are atrocious for the multimodal traces, and we do not discuss them here. For the unimodal traces, the fits are slightly better. Fig. 6 shows quantile-quantile plots for (a) normal and (b) exponential distributions fitted to the unimodal axp7 load trace of Fig. 5(b). Neither the normal or exponential distribution correctly captures the tails of the load traces. This can be seen in the figure. The quantiles of the data grow faster than those of the normal 


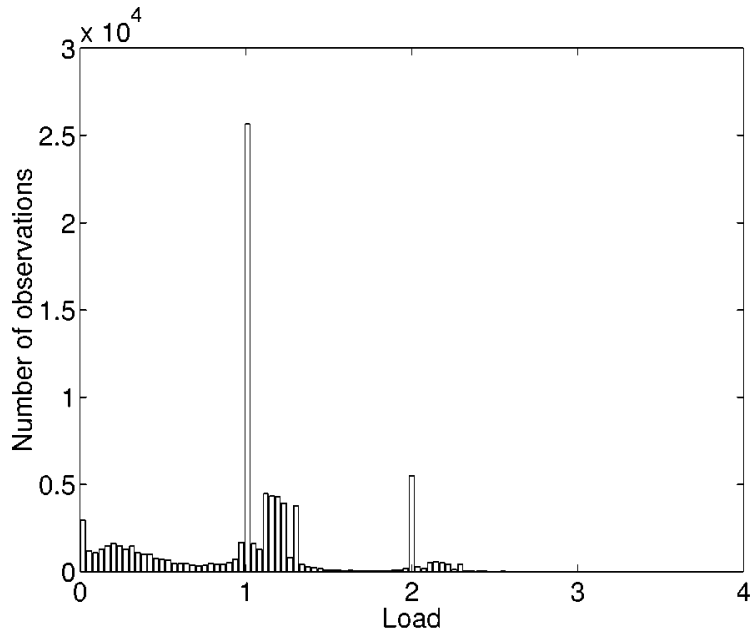

(a) $\operatorname{axp} 0$

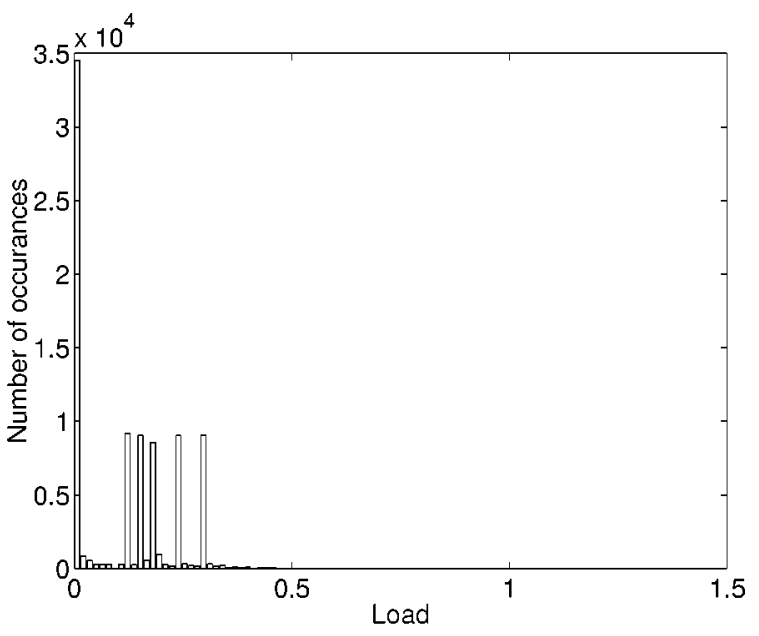

(b) $\operatorname{axp} 7$

Fig. 5. Histograms for load on axp0 and axp7 on August 19, 1997.

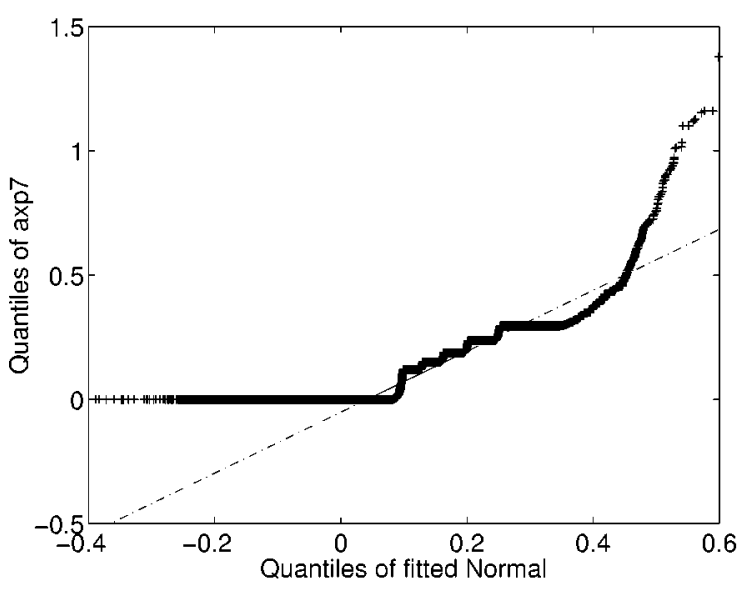

(a) $\operatorname{axp} 7$ versus normal

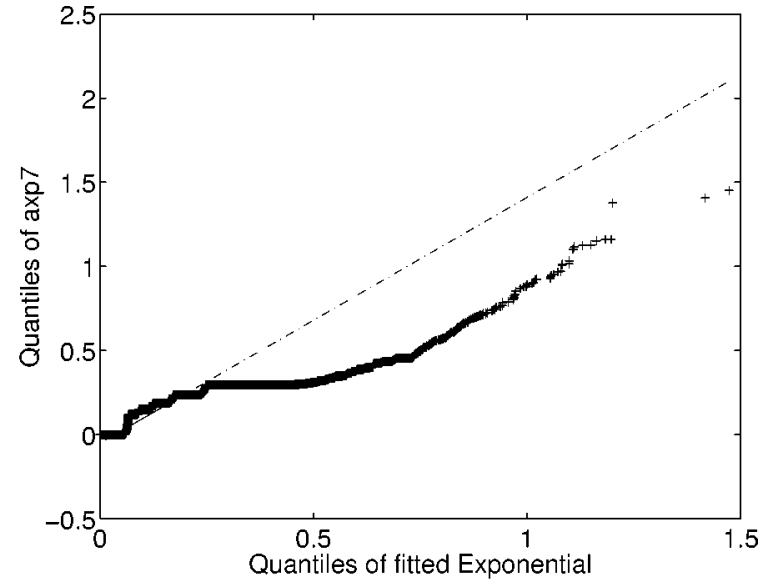

(b) $\operatorname{axp} 7$ versus exponential

Fig. 6. Quantile-quantile plots for axp7 trace of August 19, 1997.

distribution toward the right sides of Fig. 6(a). This indicates that the data has a longer or heavier tail than the normal distribution. Conversely, the quantiles of the data grow more slowly than those of the exponential distribution, as can be seen in Fig. 6(b). This indicates that the data has a shorter tail than the exponential distribution. Notice that the exponential distribution goes as $e^{-x}$ while the normal distribution goes as $e^{-x^{2}}$.

There are two implications of these complex distributions. First, simulation studies and analytic results predicated on simple, analytic distributions may produce erroneous results. Clearly, trace-driven simulation studies are to be preferred. The second implication is that prediction algorithms should not only reduce the overall variance of the load signal, but also produce errors that are better fit an analytic distribution. One reason for this is to make confidence intervals easier to compute.

\section{Time series analysis}

We examined the autocorrelation function, partial autocorrelation function, and periodogram of each of the load traces. These time series analysis tools show that past load values have a strong influence on future load values. For illustration, Fig. 7 shows (a) the axp7 load trace collected on August 19, 1997, (b) its autocorrelation function to a lag of 600 , (c) its partial au- 


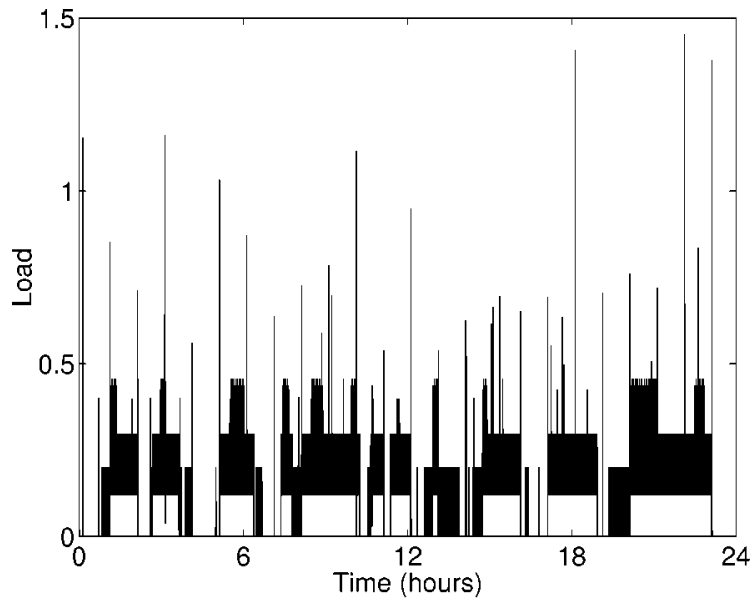

(a)

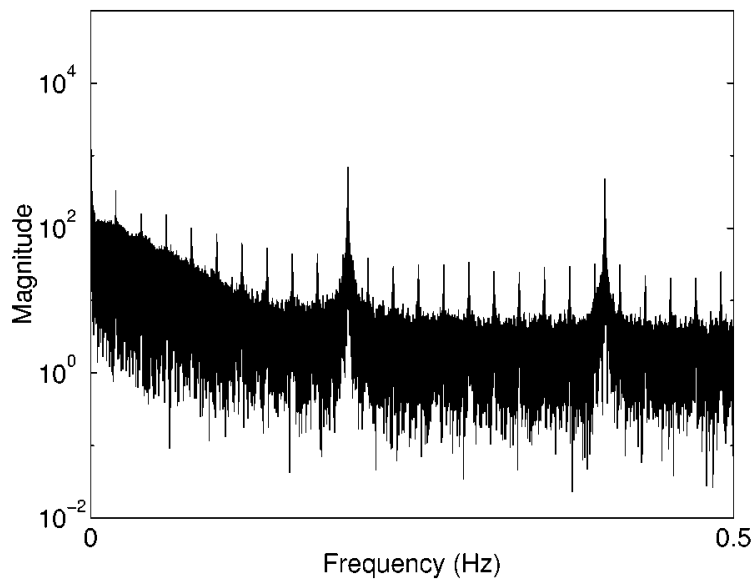

(d)

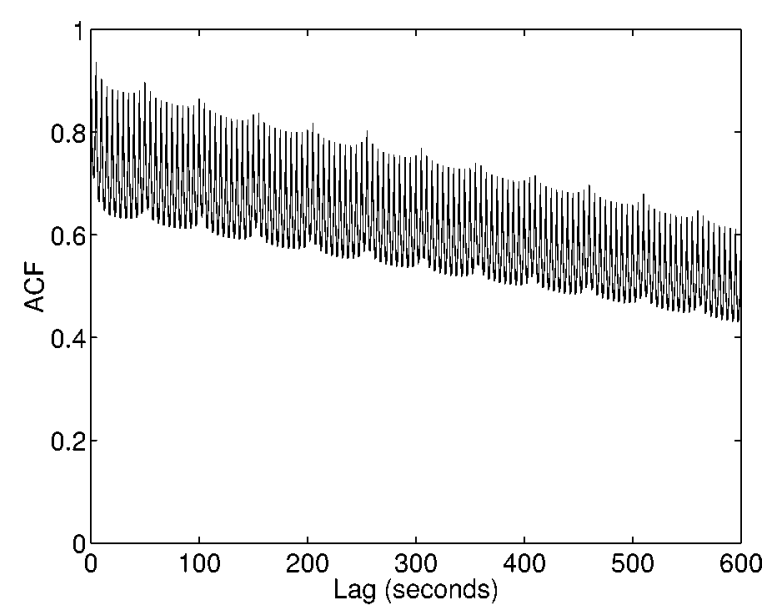

(b)

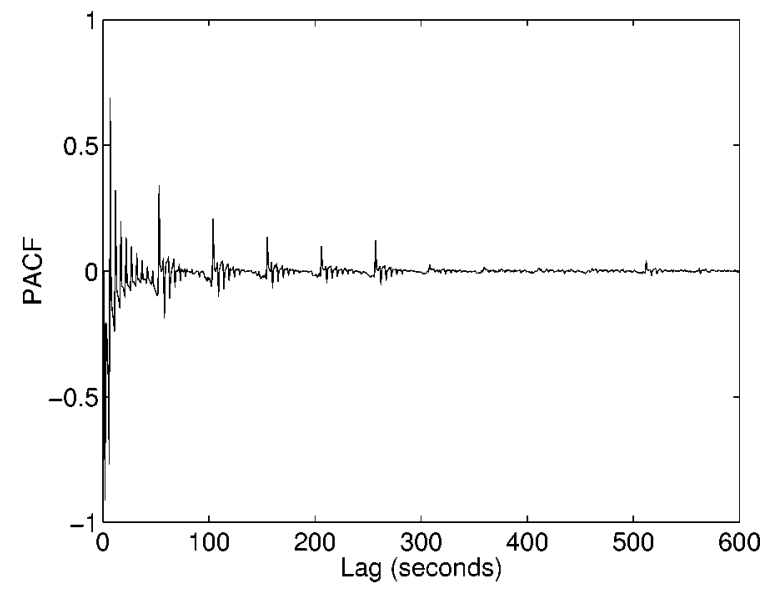

(c)

Fig. 7. Time series analysis of axp7 load trace collected on August 19, 1997: (a) load trace, (b) autocorrelation function to lag 600 (10 minutes), (c) partial autocorrelation function to lag 600 (10 minutes), (d) periodogram.

tocorrelation function to a lag of 600 , and (d) its periodogram. The analysis of this trace is representative of our results.

The autocorrelation function, which ranges from -1 to 1 , shows how well a load value at time $t$ is linearly correlated with its corresponding load value at time $t+\Delta-$ in effect, how well the value at time $t$ linearly predicts the value at time $t+\Delta$. Autocorrelation is a function of $\Delta$, and in Fig. 7(b) we show the results for $0 \leqslant \Delta \leqslant 600$. Notice that even at $\Delta=600 \mathrm{~s}$, values are still strongly correlated. This very strong, long range correlation is common to each of the load traces.

The partial autocorrelation function shows how well purely autoregressive linear models capture the correlation structure of a sequence [4], pp. 64-69. The square of the value of the function at a lag $k$ indicates the benefit of advancing from a $(k-1)$-th order model to a $k$-th order model. Intuitively, if a $k$-th order model were sufficient, then the partial autocorrelation function would be zero beyond a lag of $k$ and the autocorrelation function would be infinite. In a dual manner, if a $k$-th order purely moving average model were sufficient, then the autocorrelation function would be zero beyond a lag of $k$ and the partial autocorrelation function would be infinite. As we can see from Fig. 7(b) and (c), both functions have extremely large extents. This suggests that mixed models, which combine autoregressive and moving average components are appropriate for modelling host load.

The periodogram of a load trace is the magnitude of the Fourier transform of the load data, which we plot on a log scale (Fig. 7(d)). The periodogram shows the contribution of different frequencies (horizontal axis) to the signal. What is clear in the figure, and is true 
of all of the load traces, is that there are significant contributions from all frequencies - the signal looks much like noise. We believe the two noticeable peaks to be artifacts of the kernel sample rate - the kernel is not sampling the length of the ready queue frequently enough to avoid aliasing. Only a few of the other traces exhibit the smaller peaks, but they all share the broad noise-like appearance of this trace.

There are several implications of this time series analysis. First, the existence of such strong autocorrelation implies that load prediction based on past load values is feasible. It also suggests that simulation models and analytical work that eschews this very clear dependence may be in error. Finally, the almost noiselike periodograms suggest that quite complex, possibly nonlinear models will be necessary to produce or predict load.

\section{Self-similarity}

The key observation of this section is that each of the load traces exhibits a high degree of self-similarity. This is significant for two reasons. First, it means that load varies significantly across all time-scales - it is not the case that increasing smoothing of the load quickly tames its variance. A job will have a great deal of variance in its running time regardless of how long it is. Second, it suggests that load is difficult to model and predict well. In particular, self-similarity is indicative of long memory, possibly non-stationary stochastic processes such as fractional ARIMA models [12, $10,3]$, and fitting such models to data and evaluating them can be quite expensive.

Fig. 8 visually demonstrates the self similarity of the axp7 load trace. The top left graph in the figure plots the load on this machine versus time for 10 days. Each subsequent graph "zooms in" on the highlighted central $25 \%$ of the previous graph, until we reach the bottom right graph, which shows the central $60 \mathrm{~s}$ of the trace. The plots are scaled to make the behavior on each time scale obvious. In particular, over longer time scales, wider scales are necessary. Intuitively, a selfsimilar signal is one that looks similar on different time scales given this rescaling. Although the behavior on the different graphs is not identical, we can clearly see that there is significant variation on all time scales.

An important point is that as we smooth the signal (as we do visually as we "zoom out" toward the top of the page in Fig. 8), the load signal strongly resists becoming uniform. This suggests that low frequency components are significant in the overall mix of the signal, or, equivalently, that there is significant long range dependence. It is this property of self-similar signals that most strongly differentiates them and causes significant modeling difficulty.

Self-similarity is more than intuition - it is a well defined mathematical statement about the relationship of the autocorrelation functions of increasingly smoothed versions of certain kinds of long-memory stochastic processes. These stochastic processes model the sort of the mechanisms that give rise to self-similar signals. We shall avoid a mathematical treatment here, but interested readers may want to consult [19] or [20] for a treatment in the context of networking or [2] for its connection to fractal geometry, or [3] for a treatment from a linear time series point of view. Interestingly, self-similarity has revolutionized network traffic modelling in the 1990s $[9,19,20,28]$.

The degree and nature of the self-similarity of a sequence is summarized by the Hurst parameter, $H$ [13]. Intuitively, $H$ describes the relative contribution of low and high frequency components to the signal. Consider Fig. 9(a), which plots the periodogram (the magnitude of the Fourier transform) of the axp7 load trace of August 19, 1997 on a log-log scale. In this transformed form, we can describe the trend with a line of slope $-\beta$ (meaning that the periodogram decays hyperbolically with frequency $\omega$ as $\omega^{-\beta}$ ). The Hurst parameter $H$ is then defined as $H=(1+\beta) / 2$. As we can see in Fig. 9(a), $H=0.5$ corresponds to a line of zero slope. This is the uncorrelated noise case, where all frequencies make a roughly equal contribution. As $H$ increases beyond 0.5 , we see that low frequencies make more of a contribution. Similarly, as $H$ decreases below 0.5 , low frequencies make less of a contribution. $H>0.5$ indicates self-similarity with positive near neighbor correlation, while $H<0.5$ indicates self-similarity with negative near neighbor correlation. Fig. 9(a) shows that the axp7 trace is indeed self-similar with $H=0.875$. This method of determining the Hurst parameter is known as power spectral analysis.

Fig. 9(b) illustrates another way to think about the Hurst parameter $H$. To create the figure, we binned the load trace with increasingly larger, non-overlapping bins and then plotted the relative dispersion (the standard deviation normalized by the mean) of the binned series versus the size of the bins. For example, at a bin size of 8 we averaged the first 8 samples of the original series to form the first sample of the binned series, the next 8 to form the second sample, and so on. The 


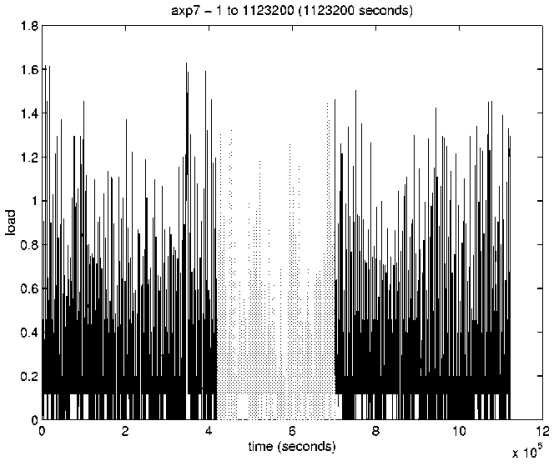

10 days

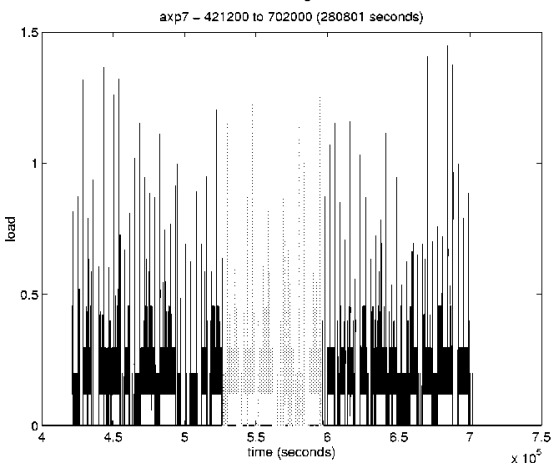

2.5 days

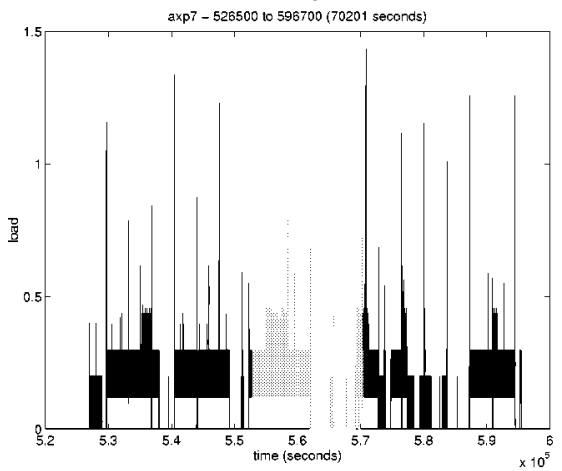

16 hours

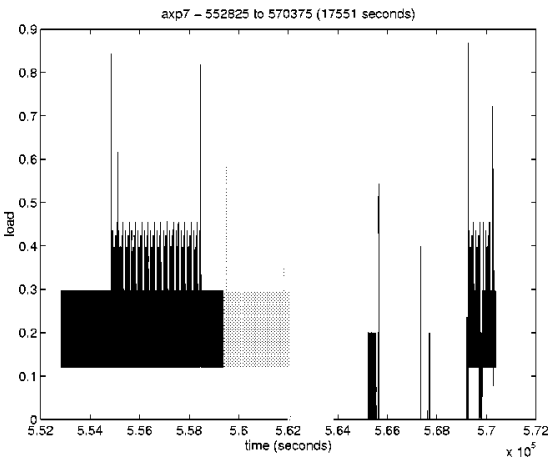

4 hours

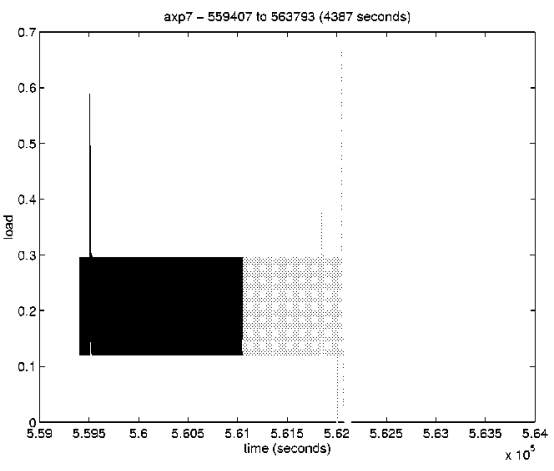

64 minutes

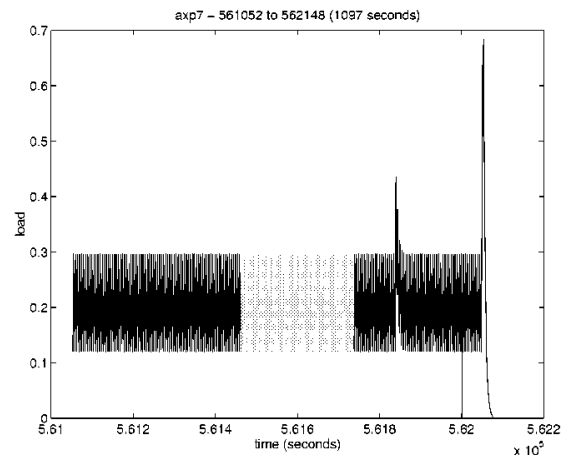

16 minutes

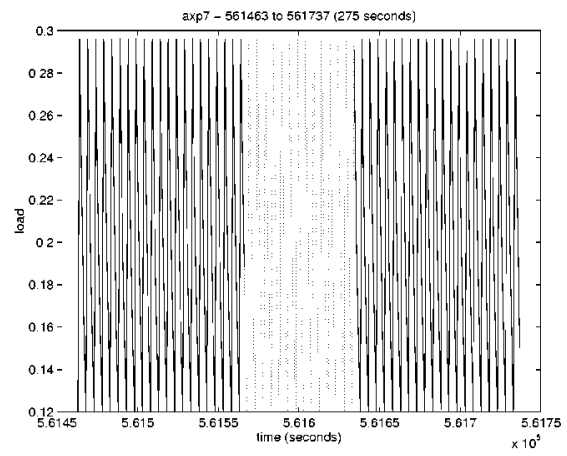

4 minutes

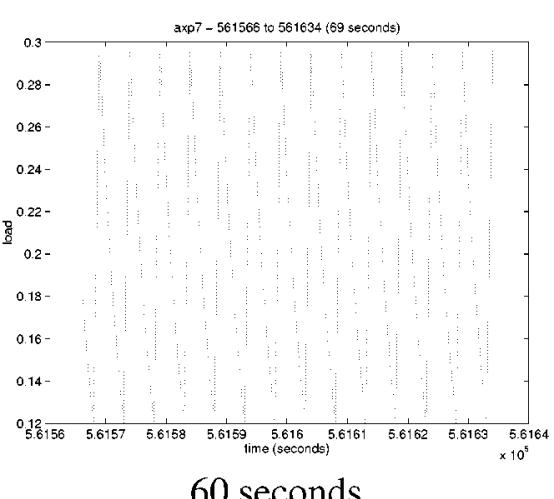

Fig. 8. Visual representation of self-similarity. Each graph plots load versus time and "zooms in" on the middle quarter. 


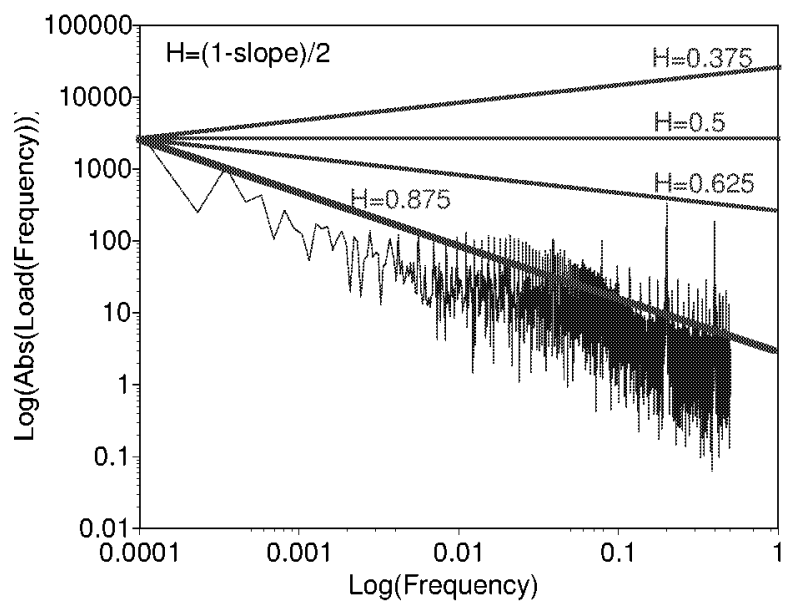

(a)

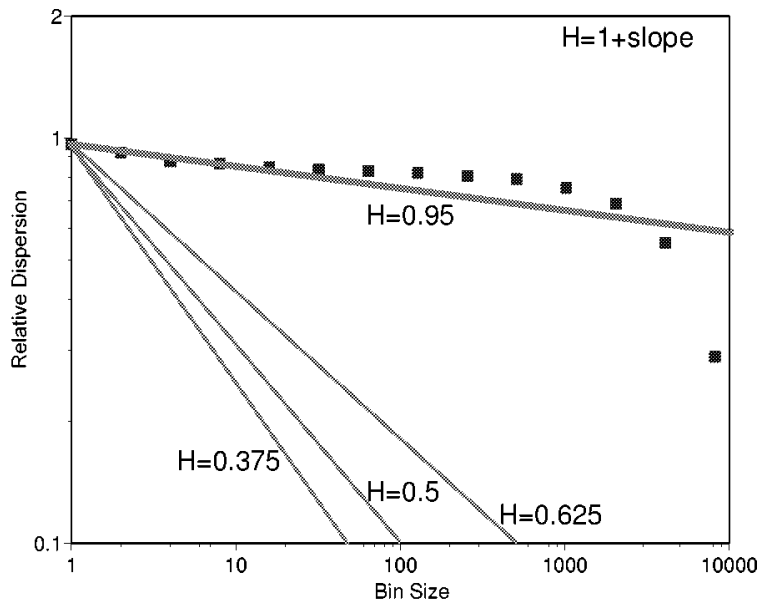

(b)

Fig. 9. Meaning of the Hurst parameter: (a) frequency domain interpretation using power spectral analysis, (b) effect of increased smoothing using dispersional analysis.

figure shows that the relative dispersion of this new 8binned series is slightly less than one. If the original load trace is self-similar, the relative dispersion should decline hyperbolically with increasing bin size. On a $\log -\log$ scale such we use in the figure this relationship would appear to be linear with a slope of $H-1$. We see that this is indeed the case for the axp7 trace. Notice that this method for estimating $H$, which is called dispersional analysis, gives a slightly different $H(0.95)$ than power spectral analysis. What is important is that in both figures we see a hyperbolic relationship, and that both estimates for $H$ are much larger than 0.5 .

We examined each of the load traces for selfsimilarity and estimated each one's Hurst parameter. There are many different estimators for the Hurst parameter [27], but there is no consensus on how to best estimate the Hurst parameter of a measured series. The most common technique is to use several Hurst parameter estimators and try to find agreement among them. The four Hurst parameter estimators we used were R/S analysis, the variance-time method, dispersional analysis, and power spectral analysis. A description of these estimators as well as several others may be found in [2]. The advantage of these estimators is that they make no assumptions about the stochastic process that generated the sequence. However, they also cannot provide confidence intervals for their estimates. Estimators such as the Whittle estimator [3] can provide confidence intervals, but a stochastic process model must be assumed over which an $H$ can be found that maximizes its likelihood.

We implemented the estimators using Matlab and validated each one by examining degenerate series with known $H$ and series with specific $H$ generated using the random midpoint displacement method. The dispersional analysis method was found to be rather weak for $H$ values less than about 0.8 and the power spectral analysis method gave the most consistent results.

Fig. 10 presents our estimates of the Hurst parameters of each of load traces. In the graph, each central point is the mean of the four estimates, while the outlying points are at $+/-$ one standard deviation. Fig. 11 shows the four actual point estimates for each trace. Notice that for small $H$ values, the standard deviation is high due to the inaccuracy of dispersional analysis. The important point is that the mean Hurst estimates are all significantly above the $H=0.5$ line and their $+/-$ one standard deviation points are also above the line.

The traces exhibit self-similarity with Hurst parameters ranging from 0.73 to 0.99 , with a strong bias toward the top of that range. An examination of the correlation coefficients (CCs) in Table 3 shows some surprising results. For the August, 1997 traces, the Hurst parameter is positively correlated with mean load $(\mathrm{CC}=0.45)$ and the standard deviation of load $(\mathrm{CC}=0.58)$, but is negatively correlated with the $\mathrm{max} / \mathrm{mean}$ load ratio $(\mathrm{CC}=-0.49)$. On the other hand, for the March, 1998 traces, the Hurst parameter is negatively correlated with mean load $(\mathrm{CC}=-0.30)$ and the standard deviation of load ( $\mathrm{CC}=-0.41)$, but is positively correlated with the max/mean ratio $(\mathrm{CC}=0.36)$. Furthermore, in the March, 1998 traces, we find the Hurst parameter is strongly positively cor- 


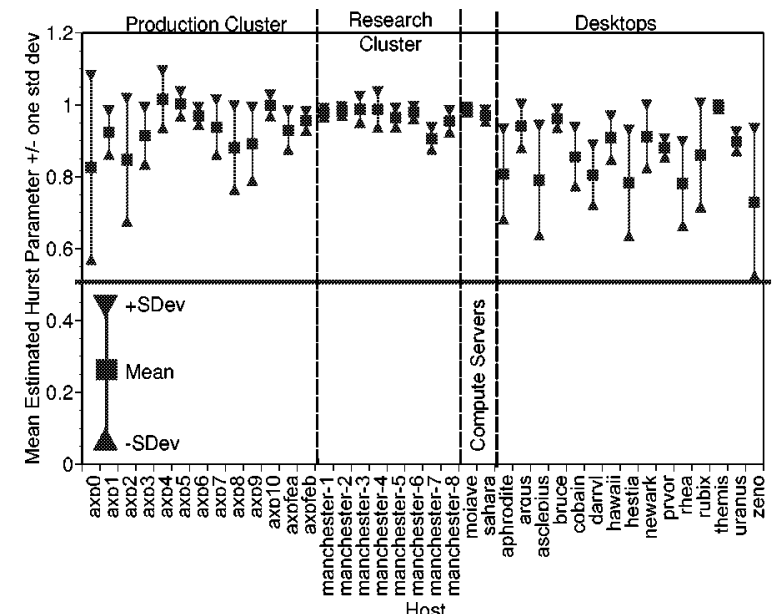

(a)

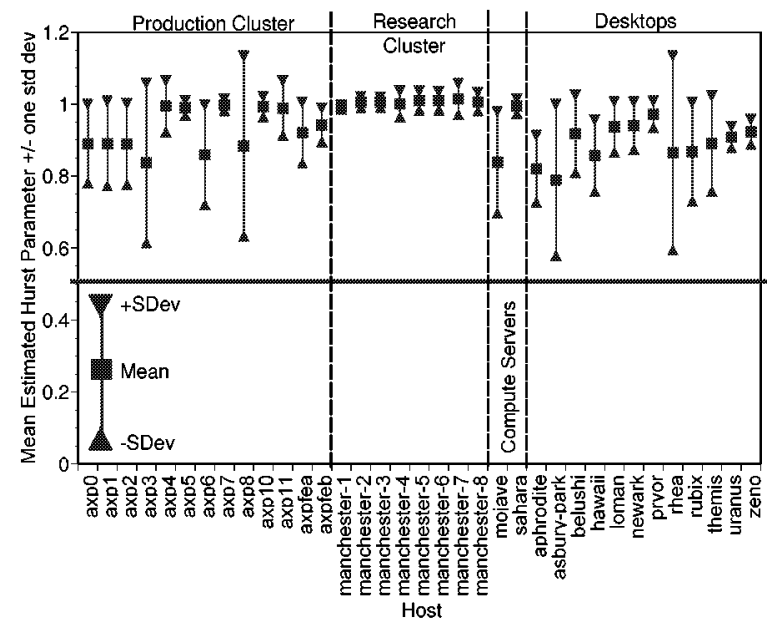

(b)

Fig. 10. Hurst parameter estimates (mean of four point estimates and standard deviation): (a) August, 1997 traces, (b) March, 1998 traces.

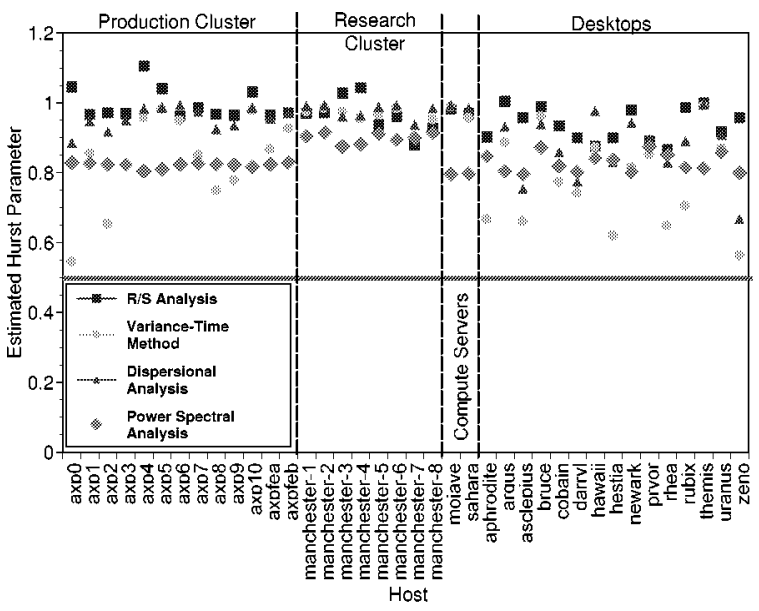

(a)

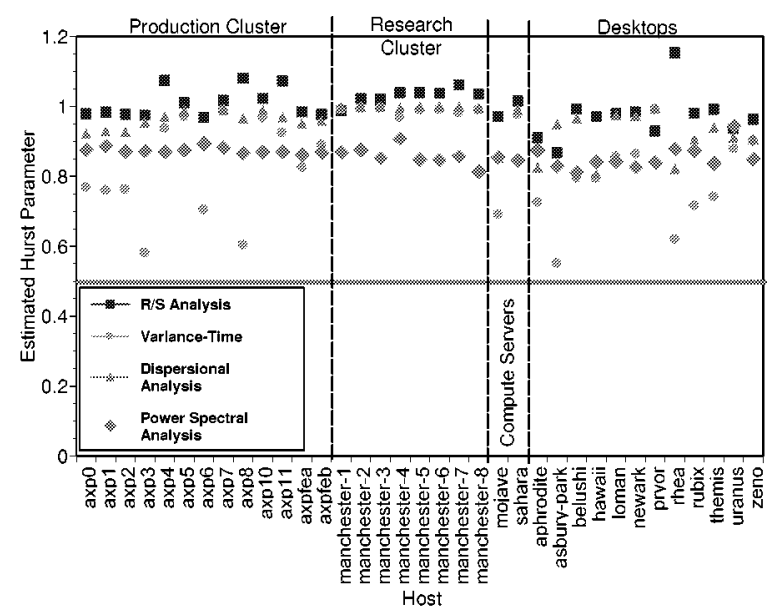

(b)

Fig. 11. Hurst parameter point estimates: (a) August, 1997 traces, (b) March, 1998 traces.

related with the epoch statistics, which is not the case at all for the August, 1997 traces. It is not clear what the cause for this difference between the traces is.

As we discussed above, self-similarity has implications for load modeling and for load smoothing. The long memory stochastic process models that can capture self-similarity tend to be expensive to fit to data and evaluate. Smoothing the load (by mapping large units of computations instead of small units, for example) may be misguided since variance may not decline with increasing smoothing intervals as quickly as quickly as expected. Consider smoothing load by averaging over an interval of length $m$. Without long range dependence $(H=0.5)$, variance would decay with $m$ as $m^{-1.0}$, while with long range dependence, as $m^{2 H-2}$ or $m^{-0.54}$ and $m^{-0.02}$ for the range of Hurst parameters we measured.

\section{Epochal behavior}

The key observation in this section is that while load changes in complex ways, the manner in which it changes remains relatively constant for relatively long periods of time. We refer to a period of time in which this stability holds true as an epoch. For example, the load signal could be a $0.25 \mathrm{~Hz}$ Sin wave for a minute and a $0.125 \mathrm{~Hz}$ sawtooth wave the next minute - each 
minute is an epoch. That these epochs exist and are long is significant because it suggests that modeling load can be simplified by modeling epochs separately from modeling the behavior within an epoch. Similarly, it suggests a two stage prediction process.

The spectrogram representation of a load trace immediately highlights the epochal behavior we discuss in this section. A spectrogram combines the frequency domain and time domain representations of a time series. It shows how the frequency domain changes locally (for a small segment of the signal) over time. For our purposes, this local frequency domain information is the "manner in which [the load] changes" to which we referred earlier. To form a spectrogram, we slide a window of length $w$ over the series, and at each offset $k$, we Fourier-transform the $w$ elements in the window to give us $w$ complex Fourier coefficients. Since our load series is real-valued, only the first $w / 2$ of these coefficients are needed. We form a plot where the $x$ coordinate is the offset $k$, the $y$ coordinate is the coefficient number, $1,2, \ldots, w / 2$ and the $z$ coordinate is the magnitude of the coefficient. To simplify presentation, we collapse to two dimensions by mapping the logarithm of the $z$ coordinate (the magnitude of the coefficient) to color. The spectrogram is basically a midpoint in the tradeoff between purely time-domain or frequencydomain representations. Along the $x$ axis we see the effects of time and along the $y$ axis we see the effects of frequency.

Fig. 12 shows a representative case, a 24 hour trace from the PSC host axp7, taken on August 19, 1997. The top graph shows the time domain representation, while the bottom graph shows the corresponding spectrogram representation. What is important to note (and which occurs in all the spectrograms of all the traces) are the relatively wide vertical bands. These indicate that the frequency domain of the underlying signal stays relatively stable for long periods of time. We refer to the width of a band as the duration of that frequency epoch.

That these epochs exist can be explained by programs executing different phases, programs being started and shut down, and the like. The frequency content within an epoch contains energy at higher frequencies because of events that happen on smaller timeframes, such as user input, device driver execution, and daemon execution.

We can algorithmically find the edges of these epochs by computing the difference in adjacent spectra in the spectrogram and then looking for those offsets where the differences exceed a threshold. Specifi- cally, we compute the sum of mean squared errors for each pair of adjacent spectra. The elements of this error vector are compared to an epsilon (5\% here) times the mean of the vector. Where this threshold is exceeded, a new epoch is considered to begin. Having found the epochs, we can examine their statistics. Fig. 13 shows the mean epoch length and the $+/$ - one standard deviation levels for each of the load traces. The mean epoch length ranges from about $150 \mathrm{~s}$ to over $450 \mathrm{~s}$, depending on which trace. The standard deviations are also relatively high ( $80 \mathrm{~s}$ to over $600 \mathrm{~s}$ ). It is the Production Cluster class which is clearly different when it comes to epoch length. The machines in this class tend to have much higher means and standard deviations than the other machines. One explanation might be that most of the machines run batch-scheduled scientific jobs which may well have longer computation phases and running times. However, two of the interactive machines also exhibit high means and standard deviations. Interestingly, there is no correlation of the mean epoch length and standard deviation to the mean load for either set of traces (Table 3 ). However, for the March, 1998 traces, we find correlations between the epoch length statistics and the Hurst parameter that are particularly strong. It is not clear why this difference exists between the traces.

The standard deviations of epoch length in Fig. 13 give us an absolute measure of the variance of epoch length. Fig. 14 shows the coefficient of variance (COV) of epoch length and mean epoch length for each trace. The COV is our relative measure of epoch length variance. Unlike with load (Section 3), these absolute and relative measures of epoch length variance are both positively correlated with the mean epoch length. In addition, the correlation is especially strong (the $\mathrm{CCs}$ are at least 0.88). As epoch length increases, it varies more in both absolute and relative terms. The statistical properties of epoch lengths are independent of the statistical properties of load.

The implication of long epoch lengths is that the problem of predicting load may be able to be decomposed into a segmentation problem (finding the epochs) and a sequence of smaller prediction subproblems (predicting load within each epoch).

Strictly speaking, epochal behavior means that load is not stationary. However, it is also not free to wander at will - clearly load cannot rise to infinite levels or fall below zero. This is compatible with the "borderline stationarity" implied by self-similarity. 

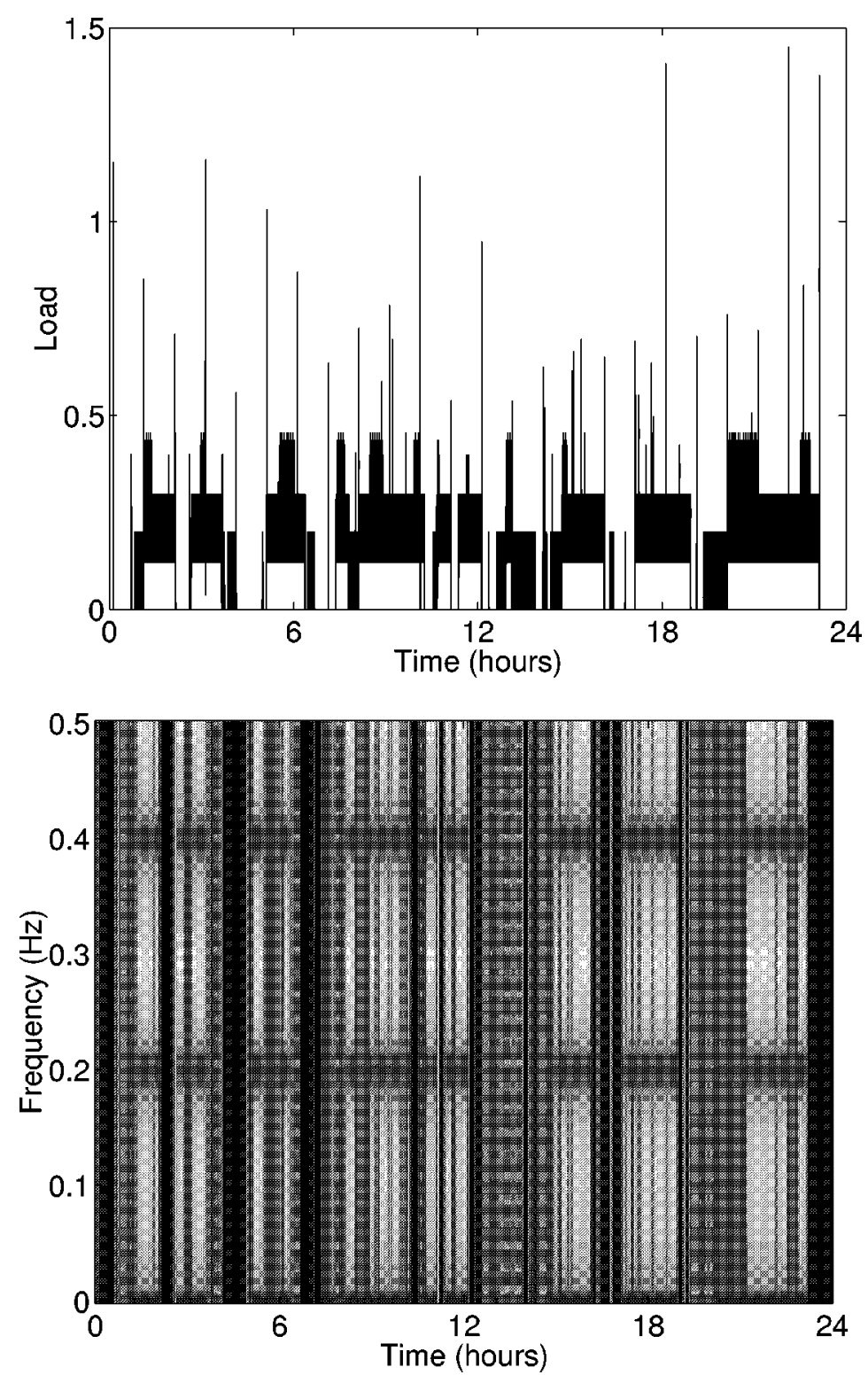

Fig. 12. Time domain and spectrogram representations of load for host axp7 on August 19, 1997.

\section{Lack of seasonality}

It is important to note that the epochal behavior of the load traces is not the same thing as seasonality in the time series analysis sense $[5,4]$. Seasonality means that there are dominant (or at least visible) underlying periodic signals on top of which are layered other signals. It is not unreasonable to expect seasonality given that other studies [21] have found that availability of compute resources to change regularly over the hours of the working day and the days of the working week.
However, examination of the power spectrums and autocorrelations of the load traces suggests that load does not exhibit seasonality. We feel this does not contradict the earlier results - the fluctuation of resources simply is not sufficiently periodic to qualify as seasonality in the strict time series sense.

\section{Conclusions and future work}

We collected long, fine grain load measurements on a wide variety of machines at two different times of the 


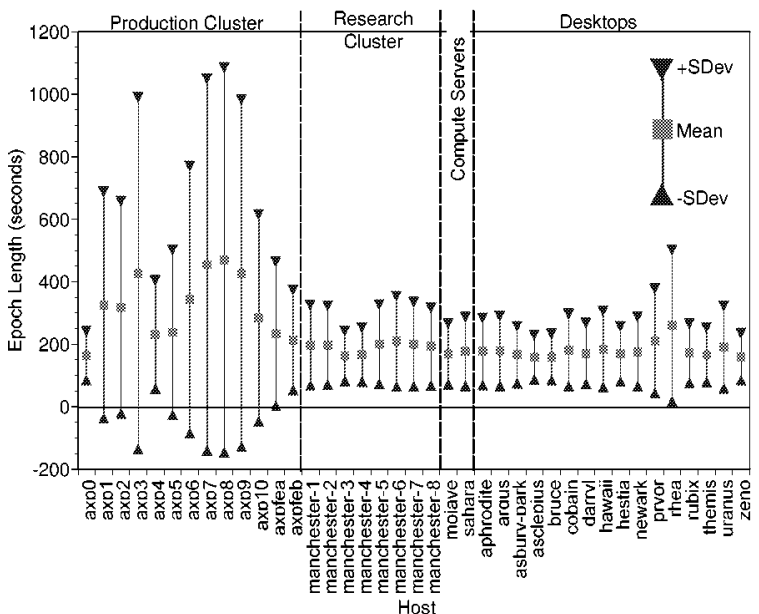

(a)

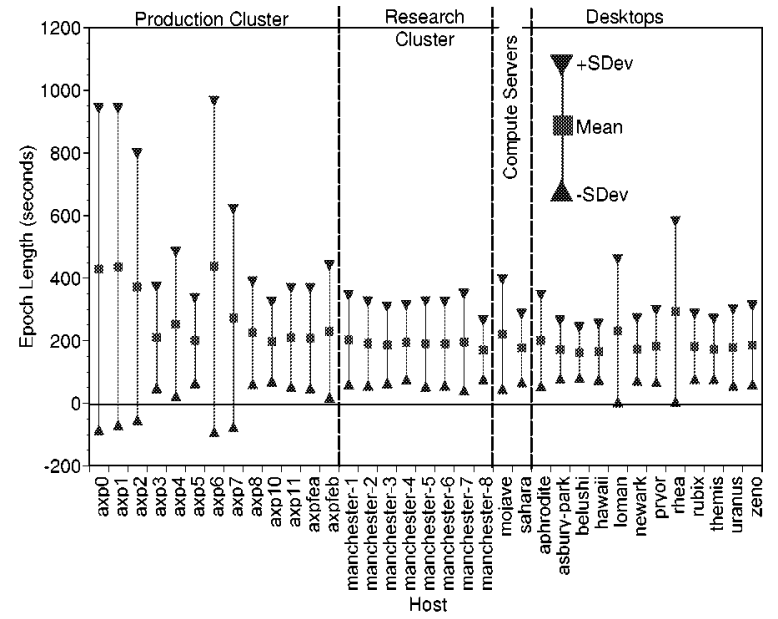

(b)

Fig. 13. Mean epoch length +/- one standard deviation: (a) August, 1997 traces, (b) March, 1998 traces.

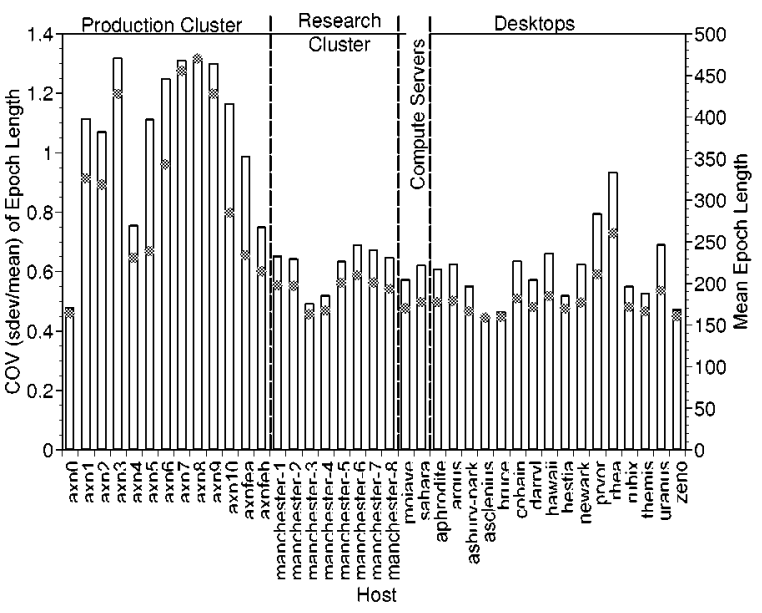

(a)

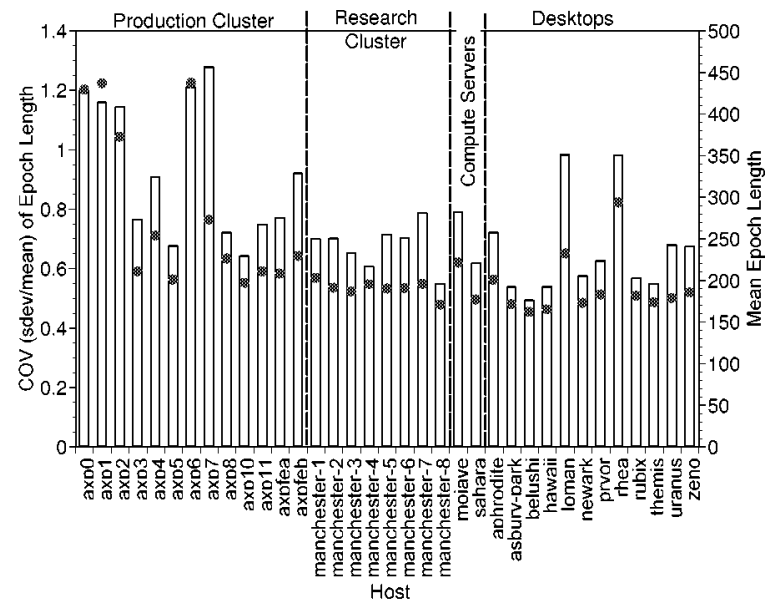

(b)

Fig. 14. COV of epoch length and mean epoch length: (a) August, 1997 traces, (b) March, 1998 traces.

year. The results of an extensive statistical analysis of these traces and their implications are the following:

(1) The traces exhibit low means but very high standard deviations and maximums. This implies that these machines have plenty of cycles to spare to execute jobs, but the execution time of these jobs will vary drastically.

(2) Absolute measures of variation are positively correlated with the mean while relative measures are negatively correlated. This suggests that it may not be unreasonable to map tasks to heavily loaded machines under some performance metrics.
(3) The traces have complex, rough, and often multimodal distributions that are not well fitted by analytic distributions such as the normal or exponential distributions, which are particularly inept at capturing the tail of the distribution. This implies that modeling and simulation that assumes convenient analytical load distributions may be flawed. Trace-driven simulation may be preferable.

(4) Load is strongly correlated over time, but has a broad, almost noise-like frequency spectrum. This implies that history-based load prediction schemes are feasible, but that linear methods may have difficulty. Realistic load models 
should capture this dependence, or trace-driven simulation should be used.

(5) The traces are self-similar with relatively high Hurst parameters. This means that load smoothing will decrease variance much more slowly than expected. It may be preferable to migrate tasks in the face of adverse load conditions instead of waiting for the adversity to be ameliorated over the long term. Self-similarity also suggests certain modeling approaches such as fractional ARIMA models [12,10,3] and non-linear models which can capture the selfsimilarity property.

(6) The traces display epochal behavior in that the local frequency content of the load signal remains quite stable for long periods of time and changes abruptly at the boundaries of such epochs. This suggests that the problem of predicting load may be able to be decomposed into a sequence of smaller subproblems.

Given these properties, we decided to study the performance of Box-Jenkins linear time series models [4] and fractional ARFIMA models [10,12,3] for short range prediction of host load. The more complex models do indeed tend to fit the data of a load trace better than simple models. For example, fractional ARFIMA models had as much as $40 \%$ lower mean squared error in some cases. Surprisingly, however, we found that simple autoregressive models performed sufficiently well for short range prediction [7]. While there were statistically significant differences between the models we studied, they were not sufficiently large to warrant the use of more complex models. Our recommendation is to use autoregressive models of order 16 or higher for prediction horizons of up to $30 \mathrm{~s}$.

\section{References}

[1] J. Arabe, A. Beguelin, B. Lowekamp, M.S.E. Seligman and P. Stephan, Dome: Parallel programming in a heterogeneous multi-user environment, Technical Report CMU-CS-95-137, Carnegie Mellon University, School of Computer Science, April 1995.

[2] J.B. Bassingthwaighte, D.A. Beard, D.B. Percival and G.M. Raymond, Fractal structures and processes, in: Chaos and the Changing Nature of Science and Medicine: An Introduction, D.E. Herbert, ed., AIP Conference Proceedings, no. 376, American Institute of Physics, April 1995, pp. 54-79.

[3] J. Beran, Statistical methods for data with long-range dependence, Statistical Science 7(4) (1992), 404-427.

[4] G.E.P. Box, G.M. Jenkins and G. Reinsel, Time Series Analysis: Forecasting and Control, 3rd ed., Prentice-Hall, 1994.
[5] P.J. Brockwell and R.A. Davis, Introduction to Time Series and Forecasting, Springer, 1996.

[6] P.A. Dinda, The statistical properties of host load, in: Proc. of 4th Workshop on Languages, Compilers, and Run-time Systems for Scalable Computers (LCR'98) (Pittsburgh, PA, 1998), Lecture Notes Comput. Sci., Vol. 1511, Springer, pp. 319-334. Extended version available as CMU Technical Report CMU-CSTR-98-143.

[7] P.A. Dinda and D.R. O'Hallaron, An evaluation of linear models for host load prediction, in: Proceedings of the 8th IEEE International Symposium on High Performance Distributed Computing (HPDC'99), IEEE, August 1999. Extended version available as Carnegie Mellon University Technical Report CMU-CS-TR-98-148.

[8] D.L. Eager, E.D. Lazowska and J. Zahorjan, The limited performance benefits of migrating active processes for load sharing, in: SIGMETRICS '88, May 1988, pp. 63-72.

[9] M. Garrett and W. Willinger, Analysis, modeling and generation of self-similar VBR video traffic, in: Proceedings of SIGCOMM '94, London, Sept. 1994.

[10] C.W.J. Granger and R. Joyeux, An introduction to longmemory time series models and fractional differencing, J. Time Series Analysis 1(1) (1980), 15-29.

[11] M. Harchol-Balter and A.B. Downey, Exploiting process lifetime distributions for dynamic load balancing, in: Proceedings of ACM SIGMETRICS '96, May 1996, pp. 13-24.

[12] J.R.M. Hosking, Fractional differencing, Biometrika 68(1) (1981), 165-176.

[13] H.E. Hurst, Long-term storage capacity of reservoirs, Trans. Amer. Soc. Civil Engineers 116 (1951), 770-808.

[14] R. Jain, The Art of Computer Systems Performance Analysis, Wiley, 1991.

[15] E.D. Jensen, C.D. Lock and H. Tokuda, A time-driven scheduling model for real-time operating systems, in: Proceedings of the Real-Time Systems Symposium, Febr. 1985, pp. 112-122.

[16] J.A. Kaplan and M.L. Nelson, A comparison of queueing, cluster, and distributed computing systems, Technical Report NASA TM 109025 (Revision 1), NASA Langley Research Center, June 1994

[17] J.F. Kurose and R. Chipalkatti, Load sharing in soft real-time distributed computer systems, IEEE Trans. Computers $\mathbf{C - 3 6 ( 8 )}$ (Aug. 1987), 993-1000.

[18] W.E. Leland and T.J. Ott, Load-balancing heuristics and process behavior, in: Proceedings of Performance and ACM SIGMETRICS, Vol. 14, 1986, pp. 54-69.

[19] W.E. Leland, M.S. Taqqu, W. Willinger and D.V. Wilson, On the self-similar nature of ethernet traffic, in: Proceedings of ACM SIGCOMM '93, Sept. 1993.

[20] P.R. Morin, The impact of self-similarity on network performance analysis, Technical Report Computer Science 95.495, Carleton University, Dec. 1995.

[21] M.W. Mutka and M. Livny, The available capacity of a privately owned workstation environment, Performance Evaluation 12(4) (July 1991), 269-284.

[22] Object Management Group. Realtime corba: A white paper. http://www.omg.org, December 1996. In progress. 
[23] A. Polze, G. Fohler and M. Werner, Predictable network computing, in: Proceedings of the 17th International Conference on Distributed Computing Systems (ICDCS '97), May 1997, pp. $423-431$.

[24] M. Rinard, D. Scales and M. Lam, Jade: A high-level machineindependent language for parallel programming, IEEE Computer 26(6) (June 1993), 28-38.

[25] C.E. Shannon, A mathematical theory of communication, Bell System Tech. J. 27 (1948), 379-423, 623-656.

[26] B. Siegell and P. Steenkiste, Automatic generation of parallel programs with dynamic load balancing, in: Proceedings of the Third International Symposium on High-Performance Distributed Computing, Aug. 1994, pp. 166-175.

[27] M.S. Taqqu, V. Teverovsky and W. Willinger, Estimators for long-range dependence: An empirical study, Fractals 3(4) (1995), 785-798.

[28] W. Willinger, S.T. Murad, R. Sherman and D.V. Wilson, Selfsimilarity through high-variability: Statistical analysis of ethernet lan traffic at the source level, in: Proceedings of ACM SIGCOMM '95, 1995, pp. 100-113. 

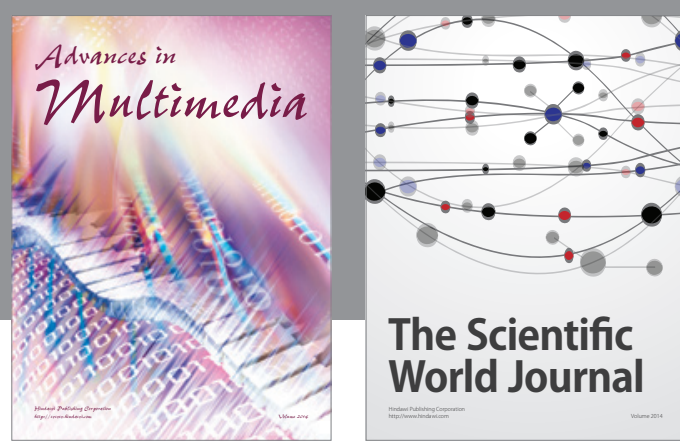

The Scientific World Journal
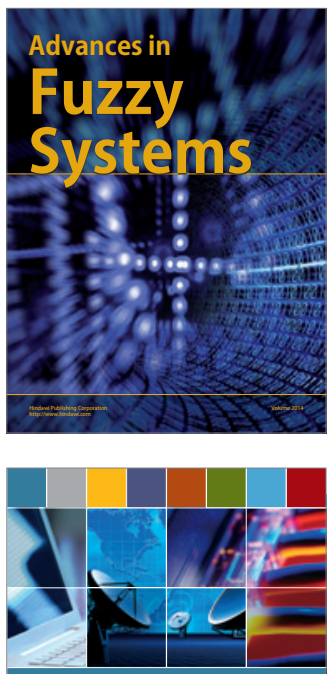

Computer Networks and Communications
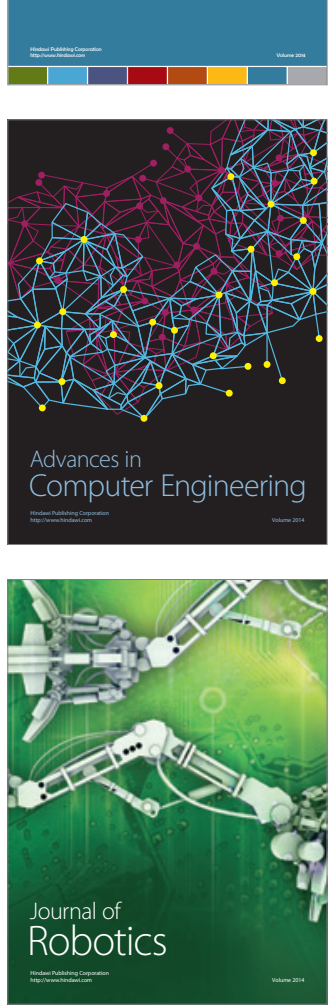
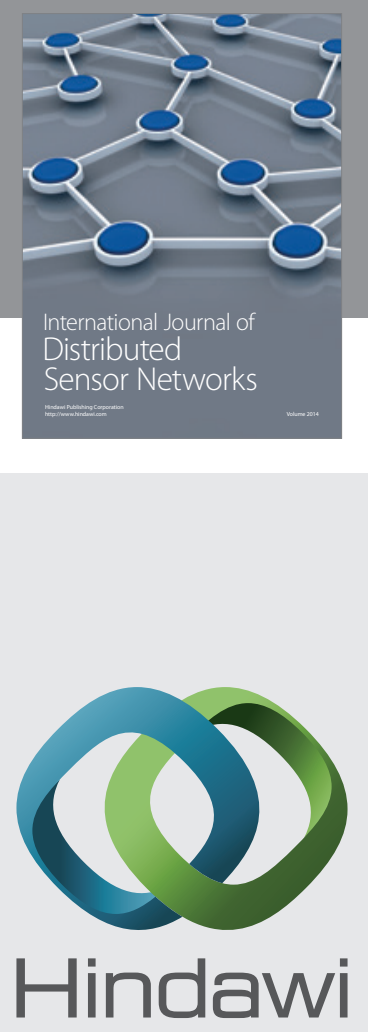

Submit your manuscripts at

http://www.hindawi.com
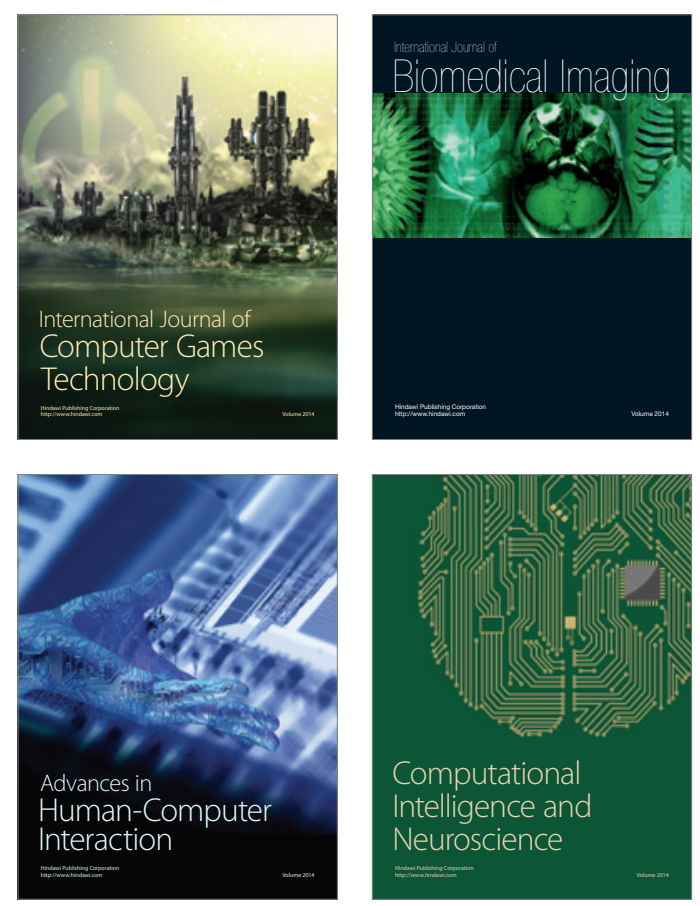
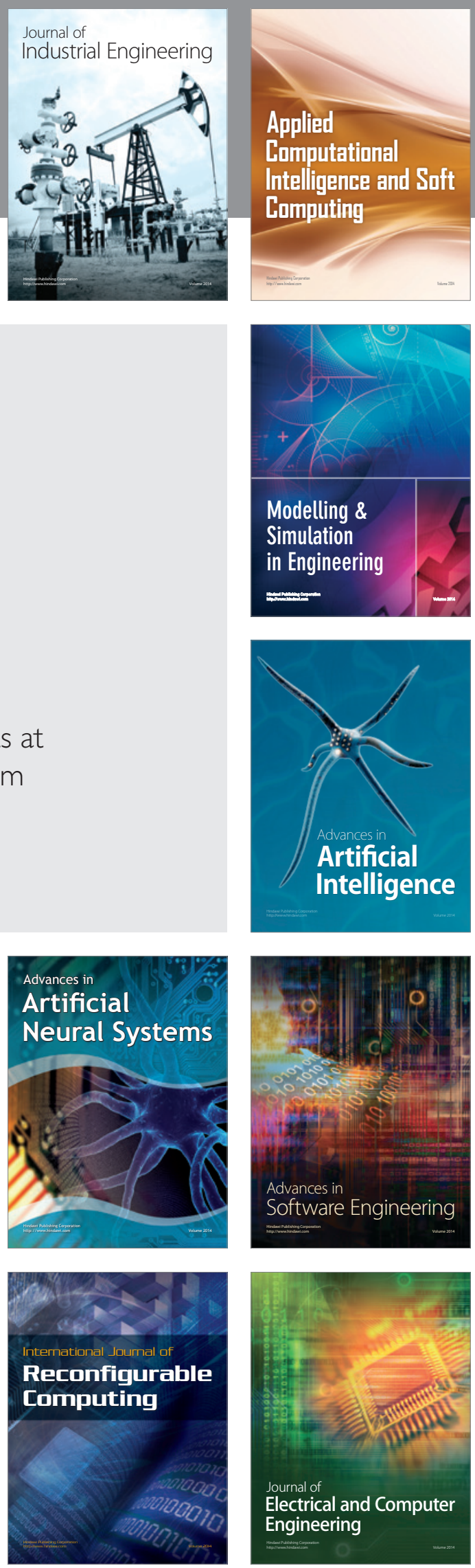